Applied and

Computational

Mathematics

Division

Computing and Applied Mathematics Laboratory

\title{
A Diffuse-Interface Description of Fluid Systems
}

D. M. Anderson and G. B. McFadden

August 1996

\section{U.S. DEPARTMENT OF COMMERCE \\ Technology Administration \\ National Institute of Standards and Technology \\ Gaithersburg, MD 20899}

$Q C$

100

.1056

N0.5887 



\section{A Diffuse-Interface Description of Fluid Systems}

\section{M. Anderson \\ G. B. MeFadden}

U.S. DEPARTMENT OF COMMERCE Technology Administration

National Institute of Standards

and Technology

Applied and Computational Mathematics Division Computing and Applied Mathematics Laboratory Gaithersburg, MD 20899

August 1996

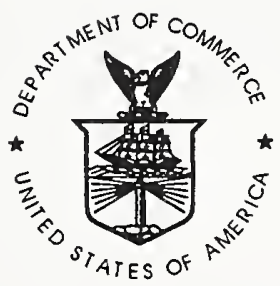

U.S. DEPARTMENT OF COMMERCE Michael Kantor, Secretary

TECHNOLOGY ADMINISTRATION

Mary L. Good, Under Secretary for Technology

NATIONAL INSTITUTE OF STANDARDS

AND TECHNOLOGY

Arati Prabnakar, Director 


\title{
A Diffuse-Interface Description of Fluid Systems
}

\author{
D. M. Anderson \\ and \\ G. B. McFadden \\ Applied and Computational Mathematics Division \\ National Institute of Standards and Technology \\ Gaithersburg, MD 20899, USA \\ tel: (301) 975-4333, fax: (301) 990-4127
}

August 1996

\begin{abstract}
We consider a diffuse-interface model for fluid-fluid systems. In classical models, an interface between two fluids is treated as infinitely thin, or sharp, and is endowed with properties such as surface tension. Diffuse-interface theories replace this sharp interface with continuous variations of an order parameter such as density in a way consistent with microscopic theories of the interface. Surface tension effects, for example, are incorporated into the model through a modified stress tensor in the classical Navier-Stokes equations. We relate the diffuse-interface model to classical, sharp interface models by deriving asymptotically the governing equations and the associated boundary conditions used in the sharp-interface formulation. We illustrate the diffuseinterface approach by modeling internal gravity waves, which have been observed experimentally by Berg et al. in xenon near its critical point. We obtain static density profiles, compute internal wave frequencies and compare with their experimental data and theoretical (classical) results both above and below the critical temperature. The results reveal a singularity in the diffuse-interface model in the limit of incompressible perturbations.
\end{abstract}




\section{Introduction}

Classical models of fluid-fluid systems treat the interfacial region between two fluids as an infinitely thin, or sharp, dividing surface and endow it with properties such as surface tension. Properties such as density take on their bulk values all the way up to the point of discontinuity. The equations of motion are solved in separate domains and the interface, where appropriate boundary conditions are applied, must be tracked explicitly.

In diffuse-interface theory, the interfacial region is represented by continuous variations of an order parameter such as density in a way consistent with microscopic theories of the interface (e.g. Rowlinson and Widom ${ }^{1}$ ). These density variations, which give the interface nonzero thickness and internal structure, connect smoothly to the bulk values of the density on either side of the interface. Interfacial excess quantities are defined in terms of the density variations through this region. Consequently, these excess quantities, rather than being defined on a two-dimensional surface, are distributed throughout a three-dimensional layer. The equations of motion, modified to account for the presence of this layer, apply over the entire domain. The interfacial region is then identified by a range of constant density contours; no interface tracking is required.

Diffuse-interface theories have been used successfully in modeling solidification and phase transitions. A notable example is the computation of dendritic growth (e.g. Kobayashi ${ }^{2}$, Wheeler et al. ${ }^{3}$ and Warren and Boettinger ${ }^{4}$ ). These models have focused on diffusive mechanisms (thermal and solutal) and have not addressed advective transport.

Recently, there have been studies of a variety of complex hydrodynamic phenomena using the diffuse-interface approach. Antanovskii ${ }^{5}$ developed a diffuse-interface model describing fluid flow, with heat and species transport for a binary fluid where composition was the order parameter. A capillary tensor, which accounted for capillary forces associated with the interfacial region, was derived using reversible thermodynamic arguments and the surface tension given in terms of the excess internal energy. This model was illustrated by computing thermocapillary-driven flow in a gap. A similar diffuse-interface model describing the motion of an isothermal binary fluid was considered by Gurtin et al. ${ }^{6}$, whose derivation was based on microforce balance laws. The model consisted of modified Navier-Stokes equations, which included a capillary tensor accounting for interfacial forces, and a coupled Cahn-Hilliard equation for the composition modified to account for hydrodynamic transport. A model for incompressible, immiscible fluids, where the order parameter was a conserved quantity transported with the fluid (e.g. density), was also described. Those authors illustrated their approach by computing the coarsening and the associated fluid motion for a binary fluid. Chella and Viñals ${ }^{7}$ used this model to compute the mixing and interfacial stretching of a binary fluid in a shear flow. Jasnow and Viñals ${ }^{8}$ studied thermocapillary drop migration and coalescence as well as spinodal decomposition using this model. In addition, they presented a derivation of the model using a Hamiltonian formalism. Jacqmin ${ }^{9}$ addressed a number of complex flows, including droplet breakup, wave-breaking and contact-line motion using the 
diffuse-interface approach. His formulation included a wall potential to model the surface energy of the container boundary. All of the above computations demonstrate the unique capabilities of the diffuse-interface approach to model flows involving complex interface morphologies and topological changes.

A situation in which diffuse-interface theory arises naturally is in the description of a fluid near its critical point. Even in the absence of complex interface shapes as described above, this situation can involve nontrivial interfacial phenomena. At temperatures below the critical point, two distinct fluid phases, separated by an interface, coexist. At temperatures above the critical point, only a single fluid phase is present; the interface ceases to exist. As the critical temperature is approached from below, the interface becomes infinitely diffuse. There has been a wealth of literature devoted to the study of the dynamics of a diffuse interface in the context of critical phenomena. Felderhof ${ }^{10}$ derived a set of equations governing the dynamics of the diffuse interface near the critical point of a pure fluid using a Lagrangian formalism. Langer and Turski ${ }^{11}$ developed a similar hydrodynamic model to describe condensation (nucleation) of a vapor near its critical point using a coarse-grained (diffuse-interface) approach. Extensive analysis using renormalization-group techniques have been performed on a diffuse-interface model ('Model H', as it is commonly known in the literature ${ }^{12}$ ) which describes the dynamics of a binary fluid phase transition as well as a pure fluid near its critical point (e.g. Halperin et al. ${ }^{13}$, Siggia et al. ${ }^{14}$, and Hohenberg and Halperin ${ }^{12}$ ). A similar mode $1^{15}$ has been used to study the dynamics of a near-critical fluid in a shear flow (Onuki and Kawasaki ${ }^{16}$ and Onuki et al. ${ }^{17}$ )

These diffuse-interface models share common features with those developed from a more computational point of view. Brackbill et al. ${ }^{18}$ developed a 'continuum surface force' model wherein they identified a volume force which represents surface tension spread over a small but finite three-dimensional interfacial domain. This volume force was related to a 'color' function which, for example, can represent density for incompressible flows. The defining characteristics of this volume force were that it gives the correct surface force in the limit of a sharp interface and is nonzero only in the interfacial region. Another related approach has been described by Unverdi and Tryggvason ${ }^{19,20}$. Their approach is a front-tracking technique which employs a numerically-diffuse description of the interface. They construct an indicator function, based on the known position of the (sharp) interface, which identifies fluid properties such as density and viscosity. This function is then artificially spread out over a small region on the scale of the computational mesh size, allowing the fluid properties to vary smoothly through this interfacial region. The surface force (i.e. surface tension) is also distributed over this interfacial region so that a single-domain approach can then be used to calculate the flow. This flow then determines how the interface is advected.

Recently, a unique hydrodynamic phenomena that occurs near a fluid's critical point has been described and analyzed by Berg et al. ${ }^{21}$ They found that, owing to its large compressibility near the critical point, xenon stratifies under its own weight and that this density stratification supports internal gravity waves when perturbed. In contrast to geophysical 
applications where length scales can be on the order of miles, the stratification in nearcritical xenon occurs on the scale of centimeters. Experimentally, they measured internal gravity wave frequencies as a function of temperature, both above and below the critical temperature. Their theoretical development consisted of two separate classical models; one that applied above the critical temperature (single-phase region) and another that applied below the critical temperature (two-phase region). In each case, they used the restricted cubic model ${ }^{22,23}$ as the equation of state.

It is in the context of critical phenomena that we shall apply the diffuse-interface approach to fluid motion. Our first objective is to formulate a diffuse-interface model for a pure fluid near its critical point. Of specific interest here will be the interpretation in terms of the underlying global quantities (mass, energy and entropy) and their associated global balance laws. Our second objective is to analyze the model in the sharp-interface limit in order to recover both the governing equations and interfacial conditions of the classical model. Our third objective is to illustrate the diffuse-interface approach using a model for internal gravity waves in xenon, which follows the work of Berg et al. ${ }^{21}$ The present approach, which applies both above and below the critical temperature, shall be compared with their experimental and theoretical results. The focus here is to use this application to highlight the diffuseinterface model rather than to improve on the good agreement between the experiments and the classical theory used by Berg et al. ${ }^{21}$ Consequently, we shall use a simpler equation of state, which allows an analytical solution for the density profile, rather than the more accurate but complicated restricted cubic model used by Berg et al.

In Section 2 we formulate the diffuse-interface model which describes the compressible, adiabatic motion of a single-component fluid near its critical point. In Section 3 we derive the classical sharp-interface governing equations and associated boundary conditions from the diffuse-interface model. In Section 4 we outline the configuration used by Berg et al. ${ }^{21}$ to study internal gravity waves in near-critical xenon. In Section 5 we obtain static density profiles using an approximate van der Waals equation of state. In Section 6 we compute internal gravity wave frequencies associated with these profiles and compare with the results of Berg et al. A discussion of the results is given in Section 7, and a conclusion is given in Section 8. We include in the appendices a derivation of the diffuse-interface equations which take into account viscous and thermal dissipation.

\section{Formulation}

The hydrodynamic equations governing inviscid, compressible flow of a single-component fluid near its critical point (e.g. Felderhof ${ }^{10}$, Langer and Turski ${ }^{11}$, Hohenberg and Halperin ${ }^{12}$, Gurtin et al. ${ }^{6}$, Jasnow and Viñals ${ }^{8}$ and $\left.\mathrm{Jacqmin}^{9}\right)$ are given by

$$
\frac{\partial \rho}{\partial t}+\nabla \cdot(\rho \boldsymbol{u})=0
$$




$$
\begin{aligned}
\rho\left(\frac{\partial \boldsymbol{u}}{\partial t}+(\boldsymbol{u} \cdot \nabla) \boldsymbol{u}\right) & =-\nabla p-\rho g \hat{z}+K \nabla \cdot \boldsymbol{T}, \\
\frac{\partial s}{\partial t}+\boldsymbol{u} \cdot \nabla s & =0
\end{aligned}
$$

where $\rho$ is the fluid density, $\boldsymbol{u}$ is the three-dimensional fluid velocity with components $(u, v, w), p=p(s, \rho)$ is the thermodynamic pressure specified by an appropriate equation of state, $s(T, \rho)$ is the entropy per unit mass, $T$ is the temperature, $g$ is the gravitational acceleration, $\hat{z}$ is the unit normal in the vertical direction, $K$ is the gradient energy coefficient which for simplicity we assume to be constant, and $\boldsymbol{T}$ is the capillary tensor given by

$$
\boldsymbol{T}=\left(\rho \nabla^{2} \rho+\frac{1}{2}|\nabla \rho|^{2}\right) \boldsymbol{I}-\nabla \rho \otimes \nabla \rho
$$

where $\boldsymbol{I}$ is the identity matrix and $\otimes$ is the tensor (outer) product. Note that $\boldsymbol{T}$ has the property

$$
\nabla \cdot \boldsymbol{T}=\rho \nabla\left(\nabla^{2} \rho\right)
$$

The key difference between these equations and the classical equations describing a twofluid system is the presence of a capillary tensor $\boldsymbol{T}$ which models capillary forces associated with the interface. Korteweg ${ }^{24}$ was the first to use such a term to describe capillary effects of a diffuse interface. The presence of this term allows these equations to apply over the entire domain, including the interfacial region; no interfacial conditions are required. Boundary conditions applied at container walls, for example, are required. These shall be discussed more thoroughly as needed for the present work. The intersection of the diffuse interface and a container boundary is, in general, a moving contact line and is not treated here but has been discussed to some extent by $\mathrm{Cahn}^{25}$ and by Jacqmin ${ }^{9}$.

In order to understand the nonclassical capillary terms appearing in the momentum equation and in the local entropy balance, it is helpful to relate equations (1) to global quantities and balance laws. We define the mass $M$, energy $E$ and entropy $S$ in a material subvolume $\Omega(t)$ of the total volume $\mathcal{V}$ by

$$
\begin{aligned}
M & =\int_{\Omega(t)} \rho d V \\
E & =\int_{\Omega(t)}\left(\frac{1}{2} \rho|\boldsymbol{u}|^{2}+\rho g z+\rho e(s, \rho)+\frac{1}{2} K|\nabla \rho|^{2}\right) d V, \\
S & =\int_{\Omega(t)} \rho s d V .
\end{aligned}
$$

The total mass is given simply by the integral over the local density. The total energy is composed of classical and nonclassical contributions. The classical terms include kinetic energy, gravitational potential energy and internal energy $e(s, \rho)$ given per unit mass. In addition to these classical contributions there is a nonclassical contribution in the form 
of a gradient energy associated with steep variations in density. ${ }^{26}$ This nonclassical term represents an energy excess associated with the interfacial region. Consistent with a constant gradient energy coefficient in equation (4b) there is no gradient entropy term in equation (4c) (e.g. see appendix B).

The governing equations (1) are consistent with the balance laws

$$
\begin{aligned}
\frac{d M}{d t} & =0 \\
\frac{d E}{d t} & =\int_{\delta \Omega(t)}\left(-p \boldsymbol{u} \cdot \hat{n}+K \hat{n} \cdot \boldsymbol{T} \cdot \boldsymbol{u}+K \frac{D \rho}{D t} \nabla \rho \cdot \hat{n}\right) d A \\
\frac{d S}{d t} & =0
\end{aligned}
$$

where $\delta \Omega(t)$ is the boundary of the subvolume and $\hat{n}$ is its outward normal. The first balance law (5a) simply represents conservation of mass. The second balance law (5b) states that the change in the energy of the subvolume is associated with the rate of (reversible) work done by pressure and capillary forces on the boundary as well as a nonclassical flux of stored energy in the interface. Note that this flux term is associated with compression of the flow in the interfacial region. Wang et al. ${ }^{27}$ identified a similar nonclassical entropy flux term in their phase-field model of solidification (see their equation (6)). Whereas their term involved the partial derivative of the order parameter with respect to time, our term involves the total derivative, $D \rho / D t=\partial \rho / \partial t+\boldsymbol{u} \cdot \nabla \rho$, since we have accounted for fluid motion. They identified this term as an entropy flux associated with variations in the phase-field at the boundary of the subvolume. The third balance law (5c) states that the change in total entropy in the subvolume $\Omega(t)$ is conserved (i.e. zero entropy production). Note that since we have neglected dissipation, these equations describe an adiabatic process.

A full derivation of the diffuse-interface equations that account for dissipative effects and for variations in the gradient energy and entropy coefficients is given in Appendix B.

\section{Sharp-Interface Limit}

Owing to the nonclassical nature of diffuse-interface models, there has been much effort devoted to relating these models to their sharp-interface counterparts. The idea behind the diffuse-interface approach is that the solutions of the diffuse-interface equations represent asymptotically the solutions of the classical, sharp-interface equations. Extensive asymptotic analyses have been performed on phase-field models of solidification to show that they recover a number of sharp-interface solidification models (e.g. see Caginalp ${ }^{28}$ ), and further comparisons between sharp- and diffuse-interface models of solidification can be found in Braun et al. ${ }^{29}$ Diffuse-interface models of fluid-fluid interfaces have received less attention in this regard. Antanovskii ${ }^{5}$ derived from the diffuse-interface model, the classical hydrostatic balance for the case of a flat interface in equilibrium and the Laplace-Young equation for the case of a spherical interface in equilibrium. Jasnow and Vinals ${ }^{8}$ derived from the capillary 
term in their momentum equation the appropriate sharp-interface tangential and normal forces when the surface tension was a slowly varying function along the interface.

In this section we derive from equations (1) the associated sharp-interface equations and boundary conditions. A complete description of the sharp-interface boundary conditions appropriate for two-phase systems can be found in Delhaye ${ }^{30}$.

We begin by writing $K=\epsilon^{2} \bar{K}=\epsilon^{2}\left(g L / \rho_{c}\right) K^{*}$ in (1) where $\epsilon$ is a small parameter measuring the thickness of the interface and $K^{*}$ is dimensionless and $O(1)$. It is clear that if we formally take the limit $\epsilon \rightarrow 0$ in the bulk regions away from the interfacial layer we recover at leading-order, the classical equations appropriate for the bulk phases,

$$
\begin{aligned}
\frac{D \rho}{D t} & =-\rho \nabla \cdot \boldsymbol{u} \\
\rho \frac{D \boldsymbol{u}}{D t} & =-\nabla p-\rho g \hat{z} \\
\frac{D s}{D t} & =0 .
\end{aligned}
$$

Next, we seek the sharp-interface boundary conditions. Since the diffuse-interface governing equations apply over the entire domain, including the interfacial region, we shall use standard pillbox arguments to obtain these conditions. We define the cylindrical pillbox as follows. We consider the contour of density upon which the interfacial region collapses in the limit $\epsilon \rightarrow 0$. The pillbox (shown in figure 1) encloses a portion of this surface at a fixed point in time in such a way that the top of the pillbox is above the surface at a height $\zeta=\delta$ and the bottom of the pillbox is below the surface at a height $\zeta=-\delta$. Here, $\zeta$ is a local coordinate which measures distance from the surface in the normal direction. We define normal vectors $\hat{n}_{t o p}, \hat{n}_{b o t}$ and $\hat{m}$ for the top, bottom, and side of the pillbox, respectively. The pillbox position is then fixed and the interface is allowed to sweep through at the next increment of time. The key limit in the pillbox argument is that $\epsilon \ll \delta \ll L$ where $L$ is an $O(1)$ length scale associated with the outer flow. In this limit, the volume of the pillbox becomes negligible on the outer scales but the variations in the density, which define the interfacial region, occur over a region fully contained within the pillbox.

Mass balance: Consider first the mass balance (1a). We integrate this equation over the volume of the pillbox $V_{p}$ to obtain

$$
0=\int_{V_{p}}\left(\frac{\partial \rho}{\partial t}+\nabla \cdot(\rho \boldsymbol{u})\right) d V .
$$

In the limit $\epsilon \ll \delta \ll L$ we have the properties that

$$
\begin{aligned}
\int_{V_{p}} \frac{\partial \rho}{\partial t} d V & \rightarrow-\int_{S_{p}} \rho \boldsymbol{u}_{I} \cdot \hat{n} d S \\
\int_{V_{p}} \frac{\partial(\rho \boldsymbol{u})}{\partial t} d V & \rightarrow-\int_{S_{p}}(\rho \boldsymbol{u}) \boldsymbol{u}_{I} \cdot \hat{n} d S
\end{aligned}
$$


where $\boldsymbol{u}_{I}=\left(0,0, w_{I}(x, y)\right)$ is the velocity of the surface described above and $S_{p}$ denotes the surface of the pillbox. Note that $\nabla \cdot \boldsymbol{u}_{I}=0$. It then follows from equation (7) and the divergence theorem that

$$
0=\int_{S_{p}} \rho\left(\boldsymbol{u}-\boldsymbol{u}_{I}\right) \cdot \hat{n}_{S} d S
$$

where $\hat{n}_{S}$ is the outward normal to the pillbox. If we further break up the surface integral into contributions from the top, bottom and side of the pillbox we find that

$$
\begin{aligned}
0= & \int_{A_{\text {top }}} \rho\left(\boldsymbol{u}-\boldsymbol{u}_{I}\right) \cdot \hat{n}_{\text {top }} d A_{\text {top }}+\int_{A_{\text {bot }}} \rho\left(\boldsymbol{u}-\boldsymbol{u}_{I}\right) \cdot \hat{n}_{\text {bot }} d A_{\text {bot }} \\
& +\oint_{C} \int_{-\delta}^{\delta} \rho\left(\boldsymbol{u}-\boldsymbol{u}_{I}\right) \cdot \hat{m} d \zeta d l
\end{aligned}
$$

where we have written the surface integral over the side of the pillbox in terms of a line integral on the surface and an integral in the normal direction. In the limit $\epsilon \ll \delta \ll L$ the contribution from the side is negligible since the integrand is bounded. Further, in this limit, $\hat{n}_{\text {top }}=-\hat{n}_{\text {bot }}=\hat{n}$ and $A_{\text {top }}=A_{\text {bot }}=A$ so that

$$
0=\left.\int_{A} \rho\left(\boldsymbol{u}-\boldsymbol{u}_{I}\right) \cdot \hat{n}\right|_{-} ^{+} d A,
$$

where $\left.\right|_{-} ^{+}$indicates the jump (top to bottom) across the interface. Since the interfacial area $A$ is arbitrary, we must have

$$
\left.\rho\left(\boldsymbol{u}-\boldsymbol{u}_{I}\right) \cdot \hat{n}\right|_{-} ^{+}=0
$$

This is the classical mass balance at an interface. Note that consistent with the original mass balance, this boundary condition allows mass flux across the interface.

Momentum balance: Consider next the momentum equation ( $1 \mathrm{~b})$. If we integrate over the pillbox as before, we find that

$$
0=\int_{V_{p}}\left\{\rho g \hat{z}+\nabla \cdot\left[\rho \boldsymbol{u} \otimes \boldsymbol{u}-\rho \boldsymbol{u} \otimes \boldsymbol{u}_{I}+p \boldsymbol{I}-K \boldsymbol{T}\right]\right\} d V
$$

where we have used equation (8b) and the fact that

$$
\rho \frac{D \boldsymbol{u}}{D t}=\frac{\partial(\rho \boldsymbol{u})}{\partial t}+\nabla \cdot(\rho \boldsymbol{u} \otimes \boldsymbol{u}) .
$$

In the limit $\epsilon \ll \delta \ll L$, the gravitational term in (13) is bounded and does not contribute. We apply the divergence theorem to the remaining terms to obtain

$$
\begin{aligned}
0 & =\int_{S_{p}}\left(\rho \boldsymbol{u} \otimes \boldsymbol{u}-\rho \boldsymbol{u} \otimes \boldsymbol{u}_{I}+p \boldsymbol{I}-K \boldsymbol{T}\right) \cdot \hat{n}_{S} d S \\
& =\int_{S_{p}}\left\{\rho \boldsymbol{u}\left(\boldsymbol{u}-\boldsymbol{u}_{I}\right) \cdot \hat{n}_{S}+p \hat{n}_{S}-K \boldsymbol{T} \cdot \hat{n}_{S}\right\} d S
\end{aligned}
$$


We break up the above surface integral into pieces evaluated on the top, bottom and sides of the pillbox. Note that in the limit $\epsilon \ll \delta \ll L$, local to the interface we have

$$
\begin{gathered}
\nabla \rho \\
\nabla^{2} \rho \sim \frac{\partial \rho}{\partial \zeta} \hat{n} \\
\frac{\partial^{2} \rho}{\partial \zeta^{2}}
\end{gathered}
$$

so that

$$
\begin{aligned}
\boldsymbol{T} \cdot \hat{n} & =\left(\rho \frac{\partial^{2} \rho}{\partial \zeta^{2}}-\frac{1}{2}\left(\frac{\partial \rho}{\partial \zeta}\right)^{2}\right) \hat{n} \\
\boldsymbol{T} \cdot \hat{m} & =\left(\rho \frac{\partial^{2} \rho}{\partial \zeta^{2}}+\frac{1}{2}\left(\frac{\partial \rho}{\partial \zeta}\right)^{2}\right) \hat{m}
\end{aligned}
$$

Since the top and bottom integrals are evaluated at $\zeta=\delta$ and $\zeta=-\delta$, respectively, and the variation in density occurs on a scale measured by $\epsilon$, we find that in the limit $\epsilon \ll \delta \ll L$ the nonclassical terms do not contribute to the top and bottom surface integrals. This leaves us with the expression

$$
\begin{aligned}
0= & \int_{A}\left(\left.\rho \boldsymbol{u}\left(\boldsymbol{u}-\boldsymbol{u}_{I}\right) \cdot \hat{n}\right|_{-} ^{+}+\left.p \hat{n}\right|_{-} ^{+}\right) d A \\
& +\oint_{C} \int_{-\delta}^{\delta}\left\{\boldsymbol{\rho} \boldsymbol{u}\left(\boldsymbol{u}-\boldsymbol{u}_{I}\right) \cdot \hat{m}+p \hat{m}-K \boldsymbol{T} \cdot \hat{m}\right\} d \zeta d l
\end{aligned}
$$

We again argue that the variation in the classical terms associated with the velocity occurs on an outer, $O(L)$, scale so that these terms do not contribute to the side integral in the limit $\delta \ll L$. It then follows that

$$
0=\int_{A}\left(\left.\rho \boldsymbol{u}\left(\boldsymbol{u}-\boldsymbol{u}_{I}\right) \cdot \hat{n}\right|_{-} ^{+}+\left.p \hat{n}\right|_{-} ^{+}\right) d A-\oint_{C} \gamma \hat{m} d l
$$

where

$$
\begin{aligned}
\gamma & =\int_{-\delta}^{\delta}\{-p+K \hat{m} \cdot \boldsymbol{T} \cdot \hat{m}\} d \zeta \\
& \sim \epsilon \int_{-\infty}^{\infty}\left\{-p+\bar{K}\left(\rho \frac{\partial^{2} \rho}{\partial \xi^{2}}+\frac{1}{2}\left(\frac{\partial \rho}{\partial \xi}\right)^{2}\right)\right\} d \xi
\end{aligned}
$$

Here $\zeta=\epsilon \xi$ and we have applied the limit $\delta / \epsilon \rightarrow \infty$. We can identify the expression for $\gamma$ as the excess Kramer's (Grand canonical) potential (see below). We next use the surface divergence theorem (e.g. Weatherburn ${ }^{31}$ )

$$
\oint_{C} \phi \hat{m} d l=\int_{A} \nabla_{S} \phi d A-\int_{A}\left(\nabla_{S} \cdot \hat{n}\right) \phi \hat{n} d A
$$


which holds for a scalar $\phi$ to further reduce equation (21). Noting that the surface area $A$ is arbitrary gives

$$
\left.\rho \boldsymbol{u}\left(\boldsymbol{u}-\boldsymbol{u}_{I}\right) \cdot \hat{n}\right|_{-} ^{+}+\left.p \hat{n}\right|_{-} ^{+}=\nabla_{S} \gamma-\left(\nabla_{S} \cdot \hat{n}\right) \gamma \hat{n} .
$$

This is the classical (inviscid) momentum jump condition across a fluid-fluid interface. Note that the first term represents an additional normal force which is nonzero whenever there is a jump in density across the interface and a nonzero mass flux. It remains to confirm that the expression for $\gamma$ given by (22) can be interpreted as the surface tension.

We appeal to the following thermodynamic arguments to obtain independently an expression for the surface tension. Consider a nonclassical extension of the free energy defined by the functional

$$
\mathcal{F}=\int_{V}\left(\rho f(\rho, T)+\frac{1}{2} K|\nabla \rho|^{2}\right) d V .
$$

An equation governing the equilibrium density profile can be obtained by minimizing this free energy subject to the constraint of constant mass. Therefore, we compute the variation

$$
0=\delta\left\{\int_{V}\left(\rho f+\frac{1}{2} K|\nabla \rho|^{2}-\lambda \rho\right) d V\right\}
$$

where $\lambda$ is a constant Lagrange multiplier. Variations in $\rho$ lead to

$$
0=f+\rho f_{\rho}-K \nabla^{2} \rho-\lambda,
$$

where $\lambda=f^{( \pm \infty)}+\rho^{( \pm \infty)} f_{\rho}^{( \pm \infty)}=\mu^{(\infty)}$ where $\mu^{(\infty)}$ is a chemical potential. This has a first integral whose form local to the surface as described above can be written

$$
\text { const. }=-\lambda \rho+\rho f-\frac{1}{2} K\left(\frac{\partial \rho}{\partial \zeta}\right)^{2} .
$$

The thermodynamic result that $p=\rho^{2} f_{\rho}$ shows that const. $=-p^{(\infty)}$.

Next, we consider the Kramer's potential energy $\Omega=\mathcal{F}-\mu^{(\infty)} M$ and define its excess by

$$
\begin{aligned}
\Omega_{x s}= & \int_{-\delta}^{a_{D}}\left\{\rho f+\frac{1}{2} K\left(\frac{\partial \rho}{\partial \zeta}\right)^{2}-\rho^{(-\infty)} f^{(-\infty)}-\mu^{(\infty)}\left(\rho-\rho^{(-\infty)}\right)\right\} d \zeta \\
& +\int_{a_{D}}^{\delta}\left\{\rho f+\frac{1}{2} K\left(\frac{\partial \rho}{\partial \zeta}\right)^{2}-\rho^{(\infty)} f^{(\infty)}-\mu^{(\infty)}\left(\rho-\rho^{(\infty)}\right)\right\} d \zeta
\end{aligned}
$$

where $a_{D}$ represents a Gibbs dividing surface and the superscripts $( \pm \infty)$ refer to the farfield values on either side of the interface. If we now use the equilibrium condition (27) to 
substitute for $\rho f$ and use $p=\rho^{2} f_{\rho}$ we find

$$
\begin{aligned}
\Omega_{x s}= & \int_{-\delta}^{a_{D}}\left\{-p+K\left(\rho \frac{\partial^{2} \rho}{\partial \zeta^{2}}+\frac{1}{2}\left(\frac{\partial \rho}{\partial \zeta}\right)^{2}\right)+\rho \lambda-\rho^{(-\infty)} f^{(-\infty)}-\mu^{(\infty)}\left(\rho-\rho^{(-\infty)}\right)\right\} d \zeta \\
& +\int_{a_{D}}^{\delta}\left\{-p+K\left(\rho \frac{\partial^{2} \rho}{\partial \zeta^{2}}+\frac{1}{2}\left(\frac{\partial \rho}{\partial \zeta}\right)^{2}\right)+\rho \lambda-\rho^{(\infty)} f^{(\infty)}-\mu^{(\infty)}\left(\rho-\rho^{(\infty)}\right)\right\} d \zeta \\
= & \int_{-\delta}^{a_{D}}\left\{-p+K\left(\rho \frac{\partial^{2} \rho}{\partial \zeta^{2}}+\frac{1}{2}\left(\frac{\partial \rho}{\partial \zeta}\right)^{2}\right)+p^{(\infty)}\right\} d \zeta \\
& +\int_{a_{D}}^{\delta}\left\{-p+K\left(\rho \frac{\partial^{2} \rho}{\partial \zeta^{2}}+\frac{1}{2}\left(\frac{\partial \rho}{\partial \zeta}\right)^{2}\right)+p^{(\infty)}\right\} d \zeta
\end{aligned}
$$

where we have used $\lambda=f^{( \pm \infty)}+\rho^{( \pm \infty)} f_{\rho}^{( \pm \infty)}=\mu^{(\infty)}$. Note that $\rho$ is the solution of equation (27); gravity does not play a role in determining the density profile local to the interface. We can combine the two integrals in equation (30) to find that $\Omega_{x s}$ is independent of the position of the dividing surface. We have,

$$
\begin{aligned}
\Omega_{x s} & =\int_{-\delta}^{\delta}\left\{-p+p^{(\infty)}+K\left(\rho \frac{\partial^{2} \rho}{\partial \zeta^{2}}+\frac{1}{2}\left(\frac{\partial \rho}{\partial \zeta}\right)^{2}\right)\right\} d \zeta \\
& =\epsilon \int_{-\infty}^{\infty}\left\{-p+\bar{K}\left(\rho \frac{\partial^{2} \rho}{\partial \xi^{2}}+\frac{1}{2}\left(\frac{\partial \rho}{\partial \xi}\right)^{2}\right)\right\} d \xi=\gamma
\end{aligned}
$$

where we have taken $p^{(\infty)}=0$ without loss of generality. This expression is identical to the previous expression (22) for $\gamma$, which confirms that the correct surface tension appears in the sharp-interface jump condition (24) derived from the diffuse-interface model. By using equations (27) and (28) this expression can be rewritten in the more familiar form

$$
\Omega_{x s}=\epsilon \bar{K} \int_{-\infty}^{\infty}\left(\frac{\partial \rho}{\partial \xi}\right)^{2} d \xi .
$$

We note here that this expression relates the gradient energy coefficient $K$ (or $\epsilon$ ) to the surface tension, which in principle can be measured experimentally.

Entropy balance: Consider next the entropy balance (1c). We can rewrite this using the mass balance $(1 \mathrm{a})$ giving

$$
0=\frac{\partial(\rho s)}{\partial t}+\nabla \cdot(\rho s \boldsymbol{u}) .
$$

Based on the same arguments that were used to derive the jump condition for mass, we find that the associated jump condition for entropy must be

$$
\left.\rho s\left(\boldsymbol{u}-\boldsymbol{u}_{I}\right) \cdot \hat{n}\right|_{-} ^{+}=\left.J s\right|_{-} ^{+}=0,
$$


where $J$ is the mass flux across the interface. This condition implies that the jump in entropy must vanish when there is nonzero mass flux across the interface.

Finally, if we impose the condition of incompressibility $\nabla \cdot \boldsymbol{u}=0$, we find that this leads to the condition $\left.\boldsymbol{u} \cdot \hat{n}\right|_{-} ^{+}=0$. Together with (12) it follows that

$$
\boldsymbol{u}^{+} \cdot \hat{n}=\boldsymbol{u}^{-} \cdot \hat{n}=\boldsymbol{u}_{I} \cdot \hat{n} .
$$

\section{A Model for Internal Gravity Waves}

We illustrate the diffuse-interface approach by modeling internal gravity waves. This application is based on results of Berg et al. ${ }^{21}$, who observed internal gravity waves in near-critical xenon. In their experiments, a mesh paddle inside a small sample of xenon was driven at a fixed frequency, stopped, and then residual internal wave frequencies were measured. This was done for a range of temperatures, both above and below the critical temperature. For the case where the cell was oriented horizontally (so that the paddle rotates about a vertical axis, see figure 2), which is the case we shall consider here, they observed two distinct internal gravity wave modes. They also modeled these waves using two separate classical models, one that applied in the one-phase region (above the critical temperature) and another that applied in the two-phase region (below the critical temperature). They used as their equation of state the restricted cubic model ${ }^{22,23}$, which gave a very accurate description of the static density profiles as the temperature varied. Their theoretical predictions for the internal gravity wave frequencies agreed well with those observed experimentally.

The model configuration consists of a rectangular cell with dimensions $0 \leq x \leq a_{x}$, $0 \leq y \leq a_{y}$ and $-L \leq z \leq L$ where gravity is in the $-z$ direction (see figure 2). A paddle is shown in the figure for reference purposes but no paddle is present in the mathematical model. The physical dimensions used by Berg et al. correspond to $a_{x}=7.6 \mathrm{~mm}, a_{y}=38 \mathrm{~mm}$ and $L=9.5 \mathrm{~mm}$. These values will be used exclusively here. The results of Berg et al. indicate that while this geometry is a simplification of the actual internal geometry of the cell used in the experiments, it is a reasonable approximation in terms of identifying the internal wave modes and frequencies.

We shall model the motion of the fluid in this cell with the diffuse-interface approach described in section 2. Our analysis of these equations shall proceed as follows. We first seek static density profiles (vertical stratification and no flow) using an approximate van der Waals equation of state. We then perturb these density profiles and identify the associated natural internal wave frequencies. We shall compare the present results with the experimental data and the theoretical predictions of Berg et al. Additional comparisons shall be made by computing the internal wave frequencies with the sharp-interface model of Berg et al. but with the equation of state used in the present work. 


\section{Basic-State Density Profiles}

The steady basic-state solution, denoted by subscript ' 0 ', has zero flow, is isothermal and horizontally uniform, and satisfies

$$
\frac{d p_{0}}{d z}=-\rho_{0} g+K \rho_{0} \frac{d^{3} \rho_{0}}{d z^{3}} .
$$

This equation determines the static density profile when an equation of state $p=p(T, \rho)$ is given. Note that since the system is isothermal, it is convenient to treat the pressure as a function of $T$ and $\rho$ rather than of $s(T, \rho)$ and $\rho$. It will be useful to work with a free energy so we employ the thermodynamic relation

$$
p=\left.\rho^{2} \frac{\partial f}{\partial \rho}\right|_{T}
$$

where $f$ is the Helmholtz free energy per unit mass. This allows us to integrate equation (36) giving

$$
K \frac{d^{2} \rho_{0}}{d z^{2}}=\frac{\partial(\rho f)}{\partial \rho}+g z+c_{0}
$$

where $c_{0}$ is a constant of integration. An equivalent derivation of equation (38) involves minimizing the total energy $E$ (equation (4b)) subject to constant total entropy $S$ (equation (4c)) and constant total mass $M$ (equation (4a)) over the total volume $\mathcal{V}$ for the case of no flow. Note that in order to focus on the critical point, we have assumed that the average density is equal to the critical density $\rho_{c}$ so that $M$ takes on the fixed value $\rho_{c} \mathcal{V}$.

We shall consider a classical van der Waals equation of state (e.g. Stanley ${ }^{32}, \mathrm{Callen}^{33}$ ) as a reasonable starting point for our analysis. Despite its shortcomings in terms of predicting the detailed behavior such as critical exponents, the van der Waals equation of state provides a simple description of the qualitative behavior of a fluid near its critical point. The van der Waals equation of state is given by

$$
\left(p+\frac{9 R T_{c}}{8 \rho_{c}} \rho^{2}\right)\left(1-\frac{\rho}{3 \rho_{c}}\right)=\rho R T
$$

where $R$ is the universal gas constant, $\rho_{c}$ is the critical density and $T_{c}$ is the critical temperature.

If we use equation (37) to write the van der Waals equation of state (39) in terms of a free energy, we find that it has a double-well structure below $T_{c}$ and a single-well structure above $T_{c}$. We shall assume a free energy per unit volume of the general form

$$
\rho f=\rho_{c} c_{1}(T)+\rho_{c} c_{2}(T)\left(\frac{\rho-\rho_{c}}{\rho_{c}}\right)+B_{0}\left[\frac{a^{2}}{2} \frac{T-T_{c}}{T_{c}}\left(\frac{\rho-\rho_{c}}{\rho_{c}}\right)^{2}+\frac{1}{4}\left(\frac{\rho-\rho_{c}}{\rho_{c}}\right)^{4}\right]
$$


where $c_{1}(T)$ is a temperature-dependent integration constant whose form is not determined by the van der Waals equation of state (39) and $c_{2}(T)$ is related to $c_{1}(T)$. The determination of $c_{1}(T)$ requires an additional thermal equation of state (e.g. see Callen ${ }^{33}$ ). Also, the parameters $B_{0}$ and $a^{2}$ are treated as adjustable. This reduces to the van der Waals form, expanded locally near the critical temperature and density, when $B_{0}=\frac{9}{16} \rho_{c} R T_{C}$ and $a^{2}=4$. Equations (38) and (40) lead to the dimensionless equation for the static density profile

$$
\tilde{\epsilon}^{2} \frac{d^{2} \tilde{\rho}}{d \tilde{z}^{2}}=\tilde{g} \tilde{z}+a^{2} \tilde{T} \tilde{\rho}+\tilde{\rho}^{3}
$$

where $\tilde{\rho}=\left(\rho-\rho_{c}\right) / \rho_{c}, \tilde{T}=\left(T-T_{c}\right) / T_{c}, \tilde{z}=z / L, \tilde{\epsilon}^{2}=K \rho_{c}^{2} / B_{0} L^{2}$ and $\tilde{g}=\rho_{c} g L / B_{0}$. We have taken $\rho=\rho_{c}$ at $z=0$. An approximate analytical solution to equation (41) can be obtained in the limit $\tilde{\epsilon} \ll 1$. For $\tilde{T}<0$ (i.e. two-phase region) and $\tilde{z}<0$, we use the method of matched asymptotic expansions to obtain

$$
\tilde{\rho}=\tilde{\rho}_{\text {out }}-a \sqrt{-\tilde{T}} \tanh \left(\frac{a \sqrt{-\tilde{T}} \tilde{z}}{\sqrt{2} \tilde{\epsilon}}\right)-a \sqrt{-\tilde{T}}+\ldots,
$$

where $\tilde{\rho}_{\text {out }}$ corresponds to the root of the equation $0=\tilde{g} \tilde{z}+a^{2} \tilde{T} \tilde{\rho}+\tilde{\rho}^{3}$ that has $\tilde{\rho}>a \sqrt{-\tilde{T}}$. For $\tilde{T}>0$ (i.e. one-phase region) we can express the solution in terms of the regular expansion

$$
\tilde{\rho}=\tilde{\rho}_{0}+\frac{6 \tilde{\epsilon}^{2} \tilde{g}^{2} \tilde{\rho}_{0}}{\left(a^{2} \tilde{T}+3 \tilde{\rho}_{0}^{2}\right)^{4}}+\ldots
$$

where $\tilde{\rho}_{0}$ is the real root of $0=\tilde{g} \tilde{z}+a^{2} \tilde{T} \tilde{\rho}_{0}+\tilde{\rho}_{0}^{3}$. Note that the density profiles are antisymmetric about $\tilde{z}=0$. Using equations (40), (37) and $s=-f_{T}$ we can compute expressions for the basic-state pressure $p_{0}(z)$ and entropy $s_{0}(z)$. Note that the entropy $s_{0}(z)$ depends on $c_{1}(T)$. Further details of this are discussed in section 7; for now we note that specification of $s_{0}(z)$ is not necessary for the development that follows.

Static density profiles are shown in figure 3 for temperatures both above and below the critical temperature. The parameter values used to calculate these profiles are $\epsilon_{D L}=10^{-4}$, $a=4.85$ and $\tilde{g}=1.631 \times 10^{-4}$ where $\epsilon_{D L}^{2}=\tilde{\epsilon}^{2} / \tilde{g}$. The parameters $a^{2}$ and $\tilde{g}$ were chosen to fit as closely as possible the density profiles shown in Berg et al. far above the critical temperature (see their figure 1). The dashed curve corresponds to the density profile at the critical temperature. Above the critical temperature, there is a single stratified phase. Below the critical temperature, the fluid separates into two stratified phases. Again, we point out that while these profiles do not embody the quantitative behavior in terms of critical exponents, they do capture the qualitative behavior of the density near the critical point.

It is interesting to note that there is a significant amount of stratification which occurs over a relatively small length scale. While this stratification often plagues those seeking to make precise measurements of physical quantities of near-critical fluids such as xenon, it can also be seen as a unique feature through which phenomena that normally occur on much larger length scales, such as those common in oceanography or atmospheric sciences, can be studied in the laboratory. 


\section{Internal Wave Frequencies}

We follow the analysis of Berg et al. ${ }^{21}$ and seek neutrally stable wave modes by introducing perturbations to the basic-state solution as follows

$$
\begin{aligned}
\rho & =\rho_{0}(z)+\hat{\rho}(z) \cos \left(q_{x} x\right) \cos \left(q_{y} y\right) e^{i \omega t} \\
u & =0+\hat{u}(z) \sin \left(q_{x} x\right) \cos \left(q_{y} y\right) e^{i \omega t} \\
v & =0+\hat{v}(z) \cos \left(q_{x} x\right) \sin \left(q_{y} y\right) e^{i \omega t} \\
w & =0+\hat{w}(z) \cos \left(q_{x} x\right) \cos \left(q_{y} y\right) e^{i \omega t} \\
p & =p_{0}(z)+\hat{p}(z) \cos \left(q_{x} x\right) \cos \left(q_{y} y\right) e^{i \omega t} \\
s & =s_{0}(z)+\hat{s}(z) \cos \left(q_{x} x\right) \cos \left(q_{y} y\right) e^{i \omega t}
\end{aligned}
$$

where $q_{x}=\pi j / a_{x}$ and $q_{y}=\pi k / a_{y}$ are the wavenumbers in the two horizontal directions with integer values for $j$ and $k$ and $\omega$ is the frequency. Note that we are interested in natural wave modes confined to the box and therefore have a discrete rather than continuous set of wave vectors. In fact, the modes we describe below have $j=k=1$. The form for the velocity components has been chosen to allow $\boldsymbol{u} \cdot \hat{n}=0$ on each wall.

As a simplification, we shall consider incompressible perturbations. In physical terms, this means that the response of a fluid parcel to a vertical perturbation is associated with changes in the background density gradient rather with any further compression of the parcel. That is, although the basic-state density profile develops as a result of the large compressibility of the fluid near its critical point, acoustic waves do not interact strongly with internal gravity waves. This can be put in more quantitative terms if we consider the Brunt-Väisälä frequency

$$
N_{B V}^{2}=-\frac{g}{\rho} \frac{\partial \rho}{\partial z}-\frac{g^{2}}{c^{2}}
$$

where $c$ is the acoustic sound speed. This quantity measures the fluid's oscillatory response to stratification and compression. ${ }^{34}$ Berg et al. argued that for the near-critical xenon system under consideration, the Brunt-Väisalä frequency could be approximated by the stratification term alone. This was based on a direct comparison for xenon of the first and second terms in equation (45). Since we expect that our density profiles approximate theirs in the bulk regions and that in the interfacial layer the density gradients used here may be quite large, we anticipate that this is a reasonable approximation to pursue here. This can be stated more formally in terms of incompressible perturbations if we consider the equation of state $p=p(s, \rho)$. It follows that

$$
\frac{D p}{D t}=\left.\frac{\partial p}{\partial s}\right|_{\rho} \frac{D s}{D t}+\left.\frac{\partial p}{\partial \rho}\right|_{s} \frac{D \rho}{D t}=\left.\frac{\partial p}{\partial s}\right|_{\rho} \frac{D s}{D t}+c^{2} \frac{D \rho}{D t} .
$$

Since the base state has no flow and varies only in the vertical direction we can reduce this 
to a statement about the perturbed quantities (denoted by primes)

$$
\left(\frac{\partial \rho^{\prime}}{\partial t}+w^{\prime} \frac{d \rho_{0}}{d z}\right)=\frac{1}{c^{2}}\left[\left(\frac{\partial p^{\prime}}{\partial t}+w^{\prime} \frac{d p_{0}}{d z}\right)-\left.\frac{\partial p}{\partial s}\right|_{\rho}\left(\frac{\partial s^{\prime}}{\partial t}+w^{\prime} \frac{d s_{0}}{d z}\right)\right] .
$$

In terms of dimensionless quantities, the condition $L g / c^{2} \ll 1$ reveals at leading order the condition of incompressible perturbations $\partial \rho^{\prime} / \partial t+w^{\prime} d \rho_{0} / d z=0$, or $\nabla \cdot \boldsymbol{u}^{\prime}=0$ using (1a).

We insert expansions (44) into the governing equations (1), use the condition of incompressible perturbations, linearize, and find that the perturbation quantities satisfy

$$
\begin{aligned}
i \omega \hat{\rho}+\hat{w} \frac{d \rho_{0}}{d z} & =0, \\
q_{x} \hat{u}+q_{y} \hat{v}+\frac{d \hat{w}}{d z} & =0, \\
i \omega \rho_{0} \hat{u}-q_{x} \hat{p} & =K \rho_{0} q_{x}\left(q^{2} \hat{\rho}-\frac{d^{2} \hat{\rho}}{d z^{2}}\right), \\
i \omega \rho_{0} \hat{v}-q_{y} \hat{p} & =K \rho_{0} q_{y}\left(q^{2} \hat{\rho}-\frac{d^{2} \hat{\rho}}{d z^{2}}\right), \\
i \omega \rho_{0} \hat{w}+\frac{d \hat{p}}{d z} & =-\hat{\rho} g+K \rho_{0}\left(-q^{2} \frac{d \hat{\rho}}{d z}+\frac{d^{3} \hat{\rho}}{d z^{3}}\right)+K \hat{\rho} \frac{d^{3} \rho_{0}}{d z^{3}}
\end{aligned}
$$

where $q^{2}=q_{x}^{2}+q_{y}^{2}$. Note that by virtue of the incompressible perturbation assumption, the equation for entropy $s$ decouples and is not needed to compute the frequency. These equations can be comvined in a straightforward manner to obtain the single perturbation equation

$$
\left[1-\frac{q^{2}}{\omega^{2}} M^{2}\right] \frac{d^{2} \hat{w}}{d z^{2}}-\left[\frac{N^{2}}{g}+\frac{q^{2}}{\omega^{2}} \frac{1}{\rho_{0}} \frac{d\left(\rho_{0} M^{2}\right)}{d z}\right] \frac{d \hat{w}}{d z}-q^{2}\left[1-\frac{N^{2}}{\omega^{2}}-\frac{q^{2}}{\omega^{2}} M^{2}\right] \hat{w}=0
$$

where

$$
\begin{aligned}
N^{2} & =-\frac{g}{\rho_{0}} \frac{d \rho_{0}}{d z} \\
M^{2} & =\frac{K}{\rho_{0}}\left(\frac{d \rho_{0}}{d z}\right)^{2} .
\end{aligned}
$$

The boundary conditions are that the vertical component of the velocity vanishes on the upper and lower boundaries $\hat{w}(L)=\hat{w}(-L)=0$. This is an eigenvalue problem where the eigenvalue is the frequency $\omega$ and the eigenfunction $\hat{w}$ characterizes the wave mode.

It is of particular interest to note that this is a second-order system. The classical model us $d$ by Berg et al. for the single-phase region, which is also second-order, can be obtained by taking $K=0$ (i.e. $M^{2}=0$ ) in equation (49). That is, the inclusion of the nonclassical terms in the incompressible limit does not result in a higher-order differential equation relative to that obtained for the classical model. However, we note here (and show later) that if we 
include the effects of compressible perturbations the result is a set of two coupled secondorder equations. We present those equations and discuss them in more detail in section 7 .

We have solved the eigenvalue problem (49) using a method given by Keller ${ }^{35}$ whereby we replace one of the homogeneous boundary conditions with an independent inhomogeneous one. The integration of the resulting inhomogeneous boundary value problem was done using SUPORT ${ }^{36}$. This code uses a superposition of linearly-independent solutions coupled with an orthonormalization procedure to maintain their (numerical) independence. The integrations were performed with either an Adams-type method or a Runge-Kutta method. The eigenvalue $\omega$ and eigenfunction $\hat{w}(z)$ which satisfy the original homogeneous boundary condition were then obtained iteratively.

Figure 4 shows the internal wave frequencies obtained experimentally (solid points) and theoretically using the restricted cubic model (dashed curves) by Berg et al. and the theoretical predictions of the present diffuse-interface approach using the van der Waals model (solid curves). The parameter values used for the diffuse-interface calculations are $\epsilon_{D L}=10^{-4}$, $a=4.85$ and $\tilde{g}=1.631 \times 10^{-4}$. The value of $\epsilon_{D L}$ used here was chosen somewhat arbitrarily but is well within the convergence region (with respect to the internal wave frequencies).

If we first focus on the experimental data, we see that there are two separate modes, whose frequencies have different behavior with respect to the temperature. Based on the eigenfunctions obtained theoretically by Berg et al., corresponding to the eigenvalues shown by the dashed curves, we know that the upper mode corresponds to a wave mode in which there is relatively large amplitude disturbances near the horizontal mid-plane of the cell. Above the critical temperature this corresponds to an internal wave set up over the whole cell, while below the critical temperature, this mode corresponds to a sloshing-type mode with relatively large interface deflection. The lower mode has relatively little motion near the center of the cell. Above the critical temperature, this corresponds to internal waves set up in both the upper and lower portions of the cell, while below the critical temperature this corresponds to a relatively quiescent lower phase and internal wave motion in the upper phase only.

There are a number of comparisons that can be made between the theoretical results predicted with the diffuse-interface model and those obtained by Berg et al. using a classical approach. While by design, the diffuse-interface model agrees with the classical theory for temperatures well above the critical temperature (recall that the parameters in the present equation of state were chosen so that the density profile matches that of the Berg et al. theory well above the critical temperature), the difference becomes more pronounced as the temperature is decreased. As we shall see below and in the next figure, this difference can be attributed in full to the difference between the two equations of state used in the two models. That is, the diffuse-interface results differ from the classical ones of Berg et al. because the variation with temperature of the density profiles obtained with the van der Waals equation of state used here does not precisely match that of the density profiles obtained using the restricted cubic model. Another result of the diffuse-interface model is 
the premature termination of the frequency curves below the critical temperature. As we shall see brlow, this is the result of a breakdown of the disturbance equation (49) as the coefficient of the term $d^{2} \hat{w} / d z^{2}$ vanishes.

Figure $\overline{5}$ shows a comparison of the frequency computed using the diffuse-interface model and that computed using the classical model of Berg et al., where in each case the equation of state (40) is used. Again the solid curves show the diffuse-interface results (with the same parameter values as shown in figure 4) and the dashed curves show the classical results. This comparison shows that with the exception of the regions below the critical temperature where the disturbance equation breaks down (see below), the diffuse-interface results reproduce the classical results. This indicates that it is the use of the simpler equation of state (40) in the present model, rather than an inherent difference between the diffuse and classical models, which accounts for the differences between the theoretical predictions shown in figure 4 .

The disturbance equation (49) is a second-order equation whose leading coefficient 1 $q^{2} M^{2} / \omega^{2}$, as noted, may vanish. Although this coefficient depends on the vertical coordinate, we can estimate the point at which it first vanishes by evaluating it at $\tilde{z}=0$, where the density gradient, and hence $M$, is largest. In terms of the frequency $\Omega=\omega / 2 \pi$ (in $\mathrm{Hz}$ ) and temperature $T$ this boundary is given by

$$
\frac{\Omega}{\sqrt{g / L}}=\frac{(q L) a^{2}}{2 \pi \sqrt{2 \tilde{g}}}\left|\frac{T-T_{c}}{T_{c}}\right| .
$$

Note that this result does not depend on the thickness of the interface. We have plotted this boundary in figure 5 (dashed-dotted line) for the parameter values as given above. This boundary is consistent with the points at which we can no longer compute numerical solutions to the present model (i.e. where the solid curves terminate in figures 4 and 5).

\section{Discussion}

In this section we discuss several of the issues raised by the above described analysis and numerical results. In particular we shall address possible improvements in terms of the equation of state used in the diffuse-interface model and also the issue of the limitations of the incompressible perturbation assumption.

The acvantage of the equation of state employed in the present analysis is that from both a physical and mathematical viewpoint it is the simplest characterization of the near-critical behavior. The disadvantage, as we have seen, is that it does not provide as accurate of a representation of the density profiles as does the restricted cubic model, for example. One possible improvement which can be made is to use a modified van der Waals equation of state (e.g. Rowlinson and Widom ${ }^{1}$, Fisk and Widom ${ }^{37}$ ). The modified van der Waals equation of state improves on the predictions in terms of critical exponents relative to the van der Waals equation of state but still allows an analytical solution for the density profiles. 
The numerical solution and analysis of the disturbance equation (49) showed that its leading coefficient can vanish. This indicates the presence of a singularity. A key assumption used to derive this equation was that the perturbations were incompressible. Although we have used a simple equation of state and have used approximate techniques to write down the basic-state density profile, it seems quite clear that a more accurate representation of the density profile shall ultimately suffer the same consequences. In order to further understand this singularity, we shall rederive the disturbance equations without making the assumption of incompressibility. Using the same approach as described in section 6, we find that the disturbance equations that retain the effects of compressibility are given by

$$
\begin{array}{r}
A_{1} \hat{p}+A_{2} \frac{d \hat{p}}{d z}+A_{3} \frac{d^{2} \hat{p}}{d z^{2}}+A_{4} \hat{q}+A_{5} \frac{d \hat{q}}{d z}+A_{6} \frac{d^{2} \hat{q}}{d z^{2}}=0, \\
B_{1} \hat{p}+B_{2} \frac{d \hat{p}}{d z}+B_{4} \hat{q}+B_{5} \frac{d \hat{q}}{d z}+B_{6} \frac{d^{2} \hat{q}}{d z^{2}}=0
\end{array}
$$

where $\hat{q}=\rho_{0} \hat{w}$ and the (variable) coefficients $A_{i}$ and $B_{i}$ are given in appendix C.

These are two coupled second-order equations describing a 'diffuse/compressible' flow. It is of interest to recover several lower-order cases from this system; that is, the 'diffuse/incompressible', 'classical/compressible' and 'classical/incompressible' cases.

The 'diffuse/incompressible' case can be obtained formally by setting $1 / c^{2}(z)=0$ in the expressions in appendix $\mathrm{C}$. In this case, the coefficients $A_{2}, A_{3}$ and $B_{2}$ all vanish. It can then be easily seen that equations (52) reduce to a second-order equation for $\hat{q}$, or equivalently, for $\hat{w}$ (see equation (49)). Therefore, in the context of the diffuse-interface formulation, the incompressible limit is singular.

This is in contrast to the classical case, as can be seen if we compare the 'classi$\mathrm{cal} /$ compressible' and 'classical/incompressible' cases. First, to obtain the classical (singlephase) description from equations (52) we take $K=0$. Here, the coefficients $A_{2}, A_{3}$ and $A_{6}$ vanish. It is again easy to see that the resulting coupled equations for $\hat{p}$ and $\hat{q}$ can be reduced to a second-order equation for $\hat{q}$. This represents the 'classical/compressible' case. In the 'classical/incompressible' case, which again we can obtain by further setting $1 / c^{2}(z)=0$, we find that the coefficient $B_{2}$ vanishes. Therefore, in the classical case, the disturbance equations are second-order regardless of whether or not the effects of compressibility are included. The above description shows that the limit of incompressible perturbations is a regular limit in the classical formulation but is a singular limit in the diffuse-interface formulation.

In order to solve equations (52), the sound speed must first be specified. This, however, cannot be calculated from the classical van der Waals equation of state alone. A thermal equation of state, consistent with the van der Waals equation, must be given (e.g. see Callen ${ }^{33}$ ).

For the present purposes, we would like only to confirm that the inclusion of the terms due to compressibility relieve the singularity observed in the incompressible calculations and allow us to calculate frequencies at lower temperatures. Therefore, we shall not be concerned with the specific form used for the sound speed. In the single-phase region, we have used 
the 'linear model' as given by Hohenberg and Barmatz ${ }^{38}$ to compute the sound speed. Note that this model is used to compute the sound speed only; we still use the van der Waals equation of state to compute the density profiles. Our calculations show that the inclusion of compressibility effects in the one-phase region lead to negligible corrections in terms of the resulting internal wave frequencies. This is to be expected based on the fact that our incompressible calculations encountered no difficulty in the one-phase region. That is, the inclusion of compressibility in the one-phase region represents a regular perturbation. This reinforces the statements given by Berg et al. ${ }^{21}$ who argued that the compressibility effects should be negligible with respect to the internal gravity wave frequency predictions. The above described 'linear model' applies in the classical sense below the critical point, in that it can be used to calculate the sound speed on either side of a sharp interface. However, there is no rigorous way of connecting the two profiles through the diffuse-interface used in the present calculations. Therefore, in the two-phase region we have used a constant value for the sound speed in order to solve the eigenvalue problem. Although this ad hoc prescription for the sound speed does not provide an accurate approximation to the true sound speed, which can vary significantly in the vertical direction, it is sufficient for our purposes in that it is nonzero everywhere and can be made to represent the true sound speed in an average sense. We have found that this approach does in fact allow us to calculate internal wave frequencies beyond the line of singularity shown in figure 5. Therefore, we can conclude that the effects of compressibility relieve this singularity and may not be negligible when using the diffuse-interface formulation as we have presented here.

As an additional check, we have calculated the solutions for the case where the capillarity terms are included in the basic-state density profile while the terms involving $M^{2}$ and its derivatives are ignored in an ad hoc way in equation (49). From the point of view of the perturbation equation, the system behaves as if it were in a single phase, with the density profile represented as before in both the one-phase and two-phase regions. The results of this calculation are graphically identical to the dashed curves shown in figure 5 , which represent the sharp-interface model results. Unlike the full incompressible case where the capillary terms $M^{2}$ are included in the perturbation equation (49), these calculations are not limited by the line of singularity.

\section{Conclusions}

We have presented diffuse-interface equations for an inviscid, compressible and adiabatic flow and have described these equations in terms of global quantities and balance laws. The key difference between the diffuse-interface formulation and the classical approach is the presence of a capillary tensor in the momentum balance. This tensor models the capillary forces associated with the diffuse interface. In contrast to the classical formulation, no interface needs to be tracked.

We have analyzed these equations and found that we can recover the classical equations 
and interfacial jump conditions for a fluid-fluid system in the sharp-interface limit. The interfacial tension is found to be consistent with the excess Kramer's (Grand canonical) potential energy.

We have used this diffuse-interface model to describe internal gravity waves in nearcritical xenon. This analysis is an extension of that done by Berg et al. ${ }^{21}$, who studied the problem experimentally and theoretically using classical hydrodynamic descriptions above and below the critical temperature. The present diffuse-interface description of the hydrodynamics can be used to calculate the internal wave frequencies both above and below the critical temperature. Our results show that the diffuse-interface model recovers the classical results when the same equation of state, used to compute the equilibrium density profiles, is used in each case.

An unexpected result identified here is that the incompressible flow limit is singular in the context of the diffuse-interface limit. That is, when the effects of compressibility are neglected, the internal wave problem is governed by a second-order perturbation equation, but when the effects of compressibility are included, the problem is governed by a system of two second-order perturbation equations. This is in contrast to the classical case where the limit of incompressibility is regular; both cases, compressible and incompressible, result in a second-order perturbation equation describing the wave modes.

\section{Acknowledgements}

The authors would like to acknowledge support by the National Aeronautics and Space Administration Microgravity Science and Applications Program. One of the authors (D.M.A.) acknowledges the support of a National Research Council Postdoctoral Fellowship. The authors would like to thank L.K. Antanovskii, R.F. Berg, S.R. Coriell, J. Lowengrub, M.J. Lyell, B.T. Murray, R.L. Pego, R.G. Rehm, R.F. Sekerka, M. Shelley and A.A. Wheeler for helpful discussions. 


\section{Appendix A. Integral Quantities}

The following expressions are used in calculating the time rates of change of the total mass, momentum, energy and entropy. First, we note that for a material volume $\Omega(t)$ which moves with the fluid the identity

$$
\frac{d}{d t} \int_{\Omega(t)} \phi d V=\int_{\Omega(t)}\left(\frac{\partial \phi}{\partial t}+\nabla \cdot(\phi \boldsymbol{u})\right) d V
$$

holds for the scalar quantity $\phi$.

Note that the following results make use of the condition of mass conservation $d M / d t=0$, or

$$
\frac{\partial \rho}{\partial t}+\nabla \cdot(\rho \boldsymbol{u})=\frac{D \rho}{D t}+\rho \nabla \cdot \boldsymbol{u}=0
$$

We find,

$$
\begin{aligned}
\frac{d}{d t} \int_{\Omega(t)} \rho \boldsymbol{u} d V & =\int_{\Omega(t)} \rho \frac{D \boldsymbol{u}}{D t} d V \\
\frac{d}{d t} \int_{\Omega(t)} \frac{1}{2} \rho|\boldsymbol{u}|^{2} d V & =\int_{\Omega(t)} \rho \boldsymbol{u} \cdot \frac{D \boldsymbol{u}}{D t} d V \\
\frac{d}{d t} \int_{\Omega(t)} \rho g z d V & =\int_{\Omega(t)} \boldsymbol{u} \cdot(\rho g \hat{z}) d V \\
\frac{d}{d t} \int_{\Omega(t)} \rho e d V & =\int_{\Omega(t)} \rho \frac{D e}{D t} d V \\
\frac{d}{d t} \int_{\Omega(t)} \frac{1}{2} K(T)|\nabla \rho|^{2} d V= & \int_{\Omega(t)}\left(K(T) \boldsymbol{T}: \nabla \boldsymbol{u}-\frac{D \rho}{D t} \nabla K(T) \cdot \nabla \rho+\frac{1}{2}|\nabla \rho|^{2} \frac{D K(T)}{D t}\right) d V \\
& +\int_{\delta \Omega(t)} K(T) \frac{D \rho}{D t} \nabla \rho \cdot \hat{n} d A
\end{aligned}
$$

\section{Appendix B. Dissipative Effects}

Here we formulate the diffuse-interface equations that account for thermal and viscous dissipation. Our approach follows the formalism used in notes by Sekerka ${ }^{39}$ on entropy production.

We start with the same total quantities for mass $M$ and energy $E$ as used in Section 2 with the exception that the gradient energy coefficient is now assumed to depend on temperature $T$. The total entropy $S$ now includes both classical and nonclassical contributions and a temperature-dependent gradient entropy coefficient. Additionally, we include a definition for total momentum $\boldsymbol{P}$. These quantities, defined on the material volume $\Omega(t)$, are

$$
M=\int_{\Omega(t)} \rho d V,
$$




$$
\begin{aligned}
\boldsymbol{P} & =\int_{\Omega(t)} \rho \boldsymbol{u} d V, \\
E & =\int_{\Omega(t)}\left(\frac{1}{2} \rho|\boldsymbol{u}|^{2}+\rho g z+\rho e(s, \rho)+\frac{1}{2} K_{E}(T)|\nabla \rho|^{2}\right) d V, \\
S & =\int_{\Omega(t)}\left(\rho s+\frac{1}{2} K_{S}(T)|\nabla \rho|^{2}\right) d V .
\end{aligned}
$$

First, we require that the mass in any subvolume $\Omega(t)$ is conserved, giving $d M / d t=0$ and

$$
\frac{\partial \rho}{\partial t}+\nabla \cdot(\rho u)=0
$$

Next, we write down the general forms of physical balance laws associated with $\boldsymbol{P}, E$ and $S$,

$$
\begin{aligned}
\frac{d \boldsymbol{P}}{d t} & =\int_{\delta \Omega(t)} \boldsymbol{m} \cdot \hat{n} d A-\int_{\Omega(t)} \rho g \hat{z} d V, \\
\frac{d E}{d t} & =\int_{\delta \Omega(t)}\left\{\boldsymbol{u} \cdot \boldsymbol{m} \cdot \hat{n}-\boldsymbol{q} \cdot \hat{n}-\boldsymbol{j}_{E}^{(n c)} \cdot \hat{n}\right\} d A, \\
\frac{d S}{d t} & +\int_{\delta \Omega(t)}\left\{\frac{\boldsymbol{q}}{T} \cdot \hat{n}+\boldsymbol{j}_{S}^{(n c)} \cdot \hat{n}\right\}=\int_{\Omega(t)} \dot{\boldsymbol{s}}^{\text {prod }} d V \geq 0,
\end{aligned}
$$

where $\boldsymbol{m}$ is a general stress tensor which may include both classical and nonclassical stresses, $\boldsymbol{q}$ is the classical conductive heat flux, $\boldsymbol{j}_{E}^{(n c)}$ is a nonclassical energy flux, $\boldsymbol{j}_{S}^{(n c)}$ is a nonclassical entropy flux and $s^{\text {prod }}$ is the volumetric rate of entropy production which may include both classical and nonclassical contributions. In physical terms, the momentum balance (B3a) states that changes in momentum result from forces on the boundary and body forces in the interior (here we are considering gravity as the only such body force). The energy balance (B3b) states that changes in energy result from the rate of work done by the forces on the boundary, the classical heat flux and a nonclassical energy flux through the boundary. The entropy balance (B3c) states that the rate of change of entropy in the control volume minus the fluxes of entropy (classical and nonclassical) through the boundary must be equal to the entropy production. The Second Law of Thermodynamics requires that this entropy production be positive for dissipative systems.

In what follows, we use the definitions (B1) to derive identities for $d \boldsymbol{P} / d t, d E / d t$ and $d S / d t$ with the aid of the results given in Appendix A. We then equate these expressions with the balance laws (B3) to obtain local balance laws in terms of the general quantities $\boldsymbol{m}, \boldsymbol{q}$, $\boldsymbol{j}_{E}^{(n c)}, \boldsymbol{j}_{S}^{(n c)}$ and $\dot{s}^{\text {prod. }}$. Finally, we appeal to the Second Law of Thermodynamics ( $\dot{s}^{\text {prod }} \geq 0$ ) to identify thermodynamically consistent forms for $\boldsymbol{m}, \boldsymbol{q}, \boldsymbol{j}_{E}^{(n c)}$ and $\boldsymbol{j}_{S}^{(n c)}$.

Momentum: We seek a local balance law for momentum by calculating the time rate of change of the total momentum given by equation (B1b) and then equating the result with the balance law for momentum (B3a). We use the divergence theorem to express boundary 
integrals as volume integrals and note that the domain of integration $\Omega(t)$ is arbitrary. This gives the local momentum balance

$$
\rho \frac{D \boldsymbol{u}}{D t}-\nabla \cdot \boldsymbol{m}+\rho g \hat{z}=0 .
$$

Energy: Next, in the same manner as described above, we obtain the local energy balance

$$
\begin{aligned}
\boldsymbol{u} \cdot\left(\rho \frac{D \boldsymbol{u}}{D t}-\nabla \cdot \boldsymbol{m}+\rho g \hat{z}\right)+\rho \frac{D e}{D t}+\nabla \cdot \boldsymbol{q}-\boldsymbol{m}: \nabla \boldsymbol{u}+K_{E}(T) \boldsymbol{T}: \nabla \boldsymbol{u} & \\
& -\frac{D \rho}{D t} \nabla K_{E} \cdot \nabla \rho+\frac{1}{2}|\nabla \rho|^{2} \frac{D K_{E}}{D t}+\nabla \cdot\left(K_{E}(T) \frac{D \rho}{D t} \nabla \rho+\boldsymbol{j}_{E}^{(n c)}\right)=0
\end{aligned}
$$

Here we have defined $\boldsymbol{T}=\left(\rho \nabla^{2} \rho+\frac{1}{2}|\nabla \rho|^{2}\right) \boldsymbol{I}-\nabla \rho \otimes \nabla \rho$, which we can identify as a Korteweglike capillary tensor ${ }^{24}$. We shall find below that the tensor $\boldsymbol{m}$ is related to $\boldsymbol{T}$. We can simplify the local energy balance by subtracting the contribution due to mechanical energy, which is expressed as the velocity $\boldsymbol{u}$ dotted with the momentum balance (B4). This leads to the simplified local energy balance

$$
\begin{aligned}
\rho \frac{D e}{D t}+\nabla \cdot \boldsymbol{q}= & \boldsymbol{m}: \nabla \boldsymbol{u}-K_{E}(T) \boldsymbol{T}: \nabla \boldsymbol{u}+\frac{D \rho}{D t} \nabla K_{E} \cdot \nabla \rho \\
& -\frac{1}{2}|\nabla \rho|^{2} \frac{D K_{E}}{D t}-\nabla \cdot\left(K_{E}(T) \frac{D \rho}{D t} \nabla \rho+j_{E}^{(n c)}\right) .
\end{aligned}
$$

We can express this equation in terms of the entropy density $s$ by first noting that the energy density $e$ is a function of $s$ and $\rho$ and then appealing to the thermodynamic relationships $\partial e / \partial s=T$ and $\partial e / \partial \rho=p / \rho^{2}$ which allow us to write

$$
\frac{D e}{D t}=T \frac{D s}{D t}+\frac{p}{\rho^{2}} \frac{D \rho}{D t} \text {. }
$$

Combining this with equations (B2) and (B6) yields

$$
\begin{aligned}
\rho T \frac{D s}{D t}+\nabla \cdot \boldsymbol{q}= & p \nabla \cdot \boldsymbol{u}+\boldsymbol{m}: \nabla \boldsymbol{u}-K_{E}(T) \boldsymbol{T}: \nabla \boldsymbol{u}+\frac{D \rho}{D t} \nabla K_{E} \cdot \nabla \rho \\
& -\frac{1}{2}|\nabla \rho|^{2} \frac{D K_{E}}{D t}-\nabla \cdot\left(K_{E}(T) \frac{D \rho}{D t} \nabla \rho+\boldsymbol{j}_{E}^{(n c)}\right) .
\end{aligned}
$$

Entropy: Finally, we can obtain a local entropy balance using equations (B1d) and (B3c). This equation is given by

$$
\begin{aligned}
\rho \frac{D s}{D t}+\frac{\nabla \cdot \boldsymbol{q}}{T}= & -K_{S}(T) \boldsymbol{T}: \nabla \boldsymbol{u}+\frac{D \rho}{D t} \nabla K_{S} \cdot \nabla \rho-\frac{1}{2}|\nabla \rho|^{2} \frac{D K_{S}}{D t} \\
& -\boldsymbol{q} \cdot \nabla\left(\frac{1}{T}\right)+\dot{s}^{\text {prod }}-\nabla \cdot\left(K_{S}(T) \frac{D \rho}{D t} \nabla \rho+\boldsymbol{j}_{S}^{(n c)}\right) .
\end{aligned}
$$

We assume that $m$ can be written as the sum of a purely classical part, which we take to be the stress tensor $\sigma=-p \boldsymbol{I}+\boldsymbol{\tau}$ where $\boldsymbol{\tau}$ is the deviatoric stress tensor, and a nonclassical 
part $\boldsymbol{m}^{(n c)}$, so that $\boldsymbol{m}=\boldsymbol{\sigma}+\boldsymbol{m}^{(n c)}$. As a consequence of this and equations (B8) and (B9) we obtain the following expression for the volumetric rate of entropy production

$$
\begin{aligned}
\dot{\boldsymbol{s}}^{\text {prod }}= & \boldsymbol{q} \cdot \nabla\left(\frac{1}{T}\right)+\frac{\boldsymbol{\tau}: \nabla \boldsymbol{u}}{T}+\frac{1}{T}\left(\boldsymbol{m}^{(n c)}-\left[K_{E}(T)-T K_{S}(T)\right] \boldsymbol{T}\right): \nabla \boldsymbol{u} \\
& +\frac{D \rho}{D t} \nabla \rho \cdot \frac{1}{T}\left(\nabla K_{E}-T \nabla K_{S}\right)-\frac{1}{2}|\nabla \rho|^{2} \frac{1}{T}\left(\frac{D K_{E}}{D t}-T \frac{D K_{S}}{D t}\right) \\
& +\nabla \cdot\left(K_{S}(T) \frac{D \rho}{D t} \nabla \rho+\boldsymbol{j}_{S}^{(n c)}\right)-\frac{1}{T} \nabla \cdot\left(K_{E}(T) \frac{D \rho}{D t} \nabla \rho+j_{E}^{(n c)}\right) .
\end{aligned}
$$

We can simplify the expression (B10) using the following argument which relates the gradient energy and gradient entropy coefficients. Suppose we write the total free energy density $f^{\text {tot }}$ (and similarly $e^{\text {tot }}$ and $s^{\text {tot }}$ ) as a function of not only of the classical variables but also $\alpha=\frac{1}{2}|\nabla \rho|^{2}$ as defined below

$$
\begin{aligned}
f^{t o t}(T, \rho, \alpha) & =f(T, \rho)+K_{F}(T) \frac{1}{2}|\nabla \rho|^{2}, \\
e^{t o t}(s, \rho, \alpha) & =e(s, \rho)+K_{E}(T) \frac{1}{2}|\nabla \rho|^{2}, \\
s^{t o t}(T, \rho, \alpha) & =s(T, \rho)+K_{S}(T) \frac{1}{2}|\nabla \rho|^{2} .
\end{aligned}
$$

Further, we assume that the same thermodynamic relations which hold for the bulk quantities also hold for the total quantities. In particular, we assume $f^{t o t}=e^{t o t}-T s^{t o t}$ and $s^{t o t}=$ $-f_{T}^{\text {tot }}$. Here the subscript denotes differentiation with respect to temperature while the other variables (i.e. $\rho$ and $\alpha$ ) are held fixed. Then, if we use $f=e-T s, s=-f_{T}$ we must also have the relations $K_{F}(T)=K_{E}(T)-T K_{S}(T), K_{S}(T)=-K_{F}^{\prime}(T), K_{E}(T)=K_{F}(T)-T K_{F}^{\prime}(T)$ so that $K_{S}^{\prime}(T)=-K_{F}^{\prime \prime}(T)$ and $K_{E}^{\prime}(T)=-T K_{F}^{\prime \prime}(T)=T K_{S}^{\prime}(T)$ where ' denotes the derivative with respect to temperature. These results lead to a simplified expression for the volumetric rate of entropy production

$$
\begin{aligned}
\dot{\boldsymbol{s}}^{\text {prod }}= & \boldsymbol{q} \cdot \nabla\left(\frac{1}{T}\right)+\frac{\boldsymbol{\tau}: \nabla \boldsymbol{u}}{T}+\frac{1}{T}\left(\boldsymbol{m}^{(n c)}-K_{F}(T) \boldsymbol{T}\right): \nabla \boldsymbol{u} \\
& +\nabla \cdot\left(K_{S}(T) \frac{D \rho}{D t} \nabla \rho+\boldsymbol{j}_{S}^{(n c)}\right)-\frac{1}{T} \nabla \cdot\left(K_{E}(T) \frac{D \rho}{D t} \nabla \rho+\boldsymbol{j}_{E}^{(n c)}\right) .
\end{aligned}
$$

Our next objective is to identify forms for the quantities $\boldsymbol{q}, \boldsymbol{\tau}, \boldsymbol{m}^{(n c)}, \boldsymbol{j}_{E}^{(n c)}$ and $\boldsymbol{j}_{S}^{(n c)}$ which are consistent with positive entropy production.

Since equation (B12) must hold when the nonclassical terms are absent we can first focus on the classical terms. In the classical case we can guarantee positive entropy production locally by choosing

$$
\boldsymbol{q}=k T^{2} \nabla\left(\frac{1}{T}\right)
$$

and

$$
\boldsymbol{\tau}=\mu\left(\nabla \boldsymbol{u}+\nabla \boldsymbol{u}^{T}\right)-\frac{2}{3} \mu(\nabla \cdot \boldsymbol{u}) \boldsymbol{I}
$$


where $k$ is the thermal conductivity and $\mu$ is the dynamic viscosity of the fluid. The form for $\boldsymbol{q}$ is the Fourier Law for heat conduction (e.g. see Kittel and Kroemer ${ }^{40}$ or Carslaw and Jaeger ${ }^{41}$ ). The form for the deviatoric stress tensor $\tau$ is just that for a Newtonian fluid (e.g. see Batchelor ${ }^{42}$ ).

We now consider the nonclassical contributions to the entropy production (B12). This form suggests that the surface tension is associated with the excess free energy, which is consistent with what we expect based on thermodynamic arguments (see Section 3). Further, we make the ansatz that the effects of surface tension are reversible in nature. This implies that if the effects of classical viscous and thermal dissipation are neglected, the entropy production is zero. The following specifications for $\boldsymbol{m}^{(n c)}, j_{E}^{(n c)}$ and $j_{S}^{(n c)}$,

$$
\begin{aligned}
\boldsymbol{m}^{(n c)} & =K_{F}(T) \boldsymbol{T} \\
j_{E}^{(n c)} & =-K_{E}(T) \frac{D \rho}{D t} \nabla \rho \\
j_{S}^{(n c)} & =-K_{S}(T) \frac{D \rho}{D t} \nabla \rho,
\end{aligned}
$$

are consistent with these conditions. This says that the nonclassical stress tensor $\boldsymbol{m}^{(n c)}$ corresponds to the Korteweg capillary tensor $\boldsymbol{T}$ with the coefficient associated with the gradient free energy. The nonclassical flux terms are in the direction of the density gradient and are zero if the flow is incompressible (i.e. $D \rho / D t=0$ ). These nonclassical energy and entropy fluxes are associated with the gradient internal energy and gradient entropy, respectively. A similar nonclassical entropy flux term was identified in the phase-field model of solidification derived by Wang et al..$^{27}$ (see their equation (6)). Whereas their term involved the partial derivative of the order parameter with respect to time, our term involves the total derivative, since we have accounted for fluid motion. They identified this term as an entropy flux associated with variations in the phase-field at the boundary of the subvolume.

The above prescription leads to the set of governing equations

$$
\begin{aligned}
\frac{D \rho}{D t} & =-\rho \nabla \cdot \boldsymbol{u} \\
\rho \frac{D \boldsymbol{u}}{D t} & =\nabla \cdot \boldsymbol{\sigma}-\rho g \hat{z}+\nabla \cdot\left(K_{F}(T) \boldsymbol{T}\right) \\
\rho \frac{D e}{D t} & =\boldsymbol{\sigma}: \nabla \boldsymbol{u}-\nabla \cdot \boldsymbol{q}-T K_{S}(T) \boldsymbol{T}: \nabla \boldsymbol{u}+\frac{D \rho}{D t} \nabla K_{E} \cdot \nabla \rho-\frac{1}{2}|\nabla \rho|^{2} \frac{D K_{E}}{D t} \\
\rho T \frac{D s}{D t} & =\boldsymbol{\tau}: \nabla \boldsymbol{u}-\nabla \cdot \boldsymbol{q}-T K_{S}(T) \boldsymbol{T}: \nabla \boldsymbol{u}+\frac{D \rho}{D t} \nabla K_{E} \cdot \nabla \rho-\frac{1}{2}|\nabla \rho|^{2} \frac{D K_{E}}{D t} .
\end{aligned}
$$

Note that

$$
\nabla \cdot\left(K_{F}(T) \boldsymbol{T}\right)=\rho \nabla\left(K_{F}(T) \nabla^{2} \rho\right)-\frac{1}{2}|\nabla \rho|^{2} \nabla K_{F}(T) .
$$




\section{Appendix C. Coefficients of Compressible Equations}

The following are the coefficients appearing in the compressible perturbation equations (52).

$$
\begin{aligned}
& A_{1}=\frac{1}{i \omega}\left[q^{2}-\frac{\omega^{2}}{c^{2}(z)}+K q^{2} \rho_{0}\left(\frac{q^{2}}{c^{2}(z)}-\left(\frac{1}{c^{2}(z)}\right)^{\prime \prime}\right)\right] \\
& A_{2}=-\frac{2 K q^{2}}{i \omega} \rho_{0}\left(\frac{1}{c^{2}(z)}\right)^{\prime} \\
& A_{3}=-\frac{K q^{2}}{i \omega} \frac{\rho_{0}}{c^{2}(z)} \\
& A_{4}=\frac{N_{K}(z)}{g}-\frac{K q^{4}}{g \omega^{2}} \rho_{0} N_{K}(z)+\frac{K q^{2}}{g \omega^{2}} \rho_{0} N_{K}^{\prime \prime}(z), \\
& A_{5}=1+\frac{2 K q^{2}}{g \omega^{2}} \rho_{0} N_{K}^{\prime}(z) \\
& A_{6}=\frac{K q^{2}}{g \omega^{2}} \rho_{0} N_{K}(z), \\
& B_{1}=\frac{1}{i \omega}\left[\frac{1}{\rho_{0}^{2}} \frac{d \rho_{0}}{d z}+\frac{\omega^{2}}{q^{2}}\left(\frac{1}{\rho_{0} c^{2}(z)}\right)^{\prime}+\frac{g}{\rho_{0} c^{2}(z)}-\frac{K}{\rho_{0} c^{2}(z)} \frac{d^{3} \rho_{0}}{d z^{3}}\right] \\
& B_{2}=\frac{1}{i \omega}\left[\frac{\omega^{2}}{q^{2} \rho_{0} c^{2}(z)}\right], \\
& B_{4}=-\frac{1}{g q^{2}}\left(\frac{N_{K}(z)}{\rho_{0}}\right)^{\prime}-\frac{N_{K}(z)}{\omega^{2} \rho_{0}}+\frac{1}{\rho_{0}}+\frac{K}{g \omega^{2}} \frac{N_{K}(z)}{\rho_{0}} \frac{d^{3} \rho_{0}}{d z^{3}} \\
& B_{5}=-\frac{N_{K}(z)}{g q^{2} \rho_{0}}+\frac{1}{q^{2} \rho_{0}^{2}} \frac{d \rho_{0}}{d z}, \\
& B_{6}=-\frac{1}{q^{2} \rho_{0}},
\end{aligned}
$$

where

$$
N_{K}(z)=-\frac{g}{\rho_{0}} \frac{d \rho_{0}}{d z}-\frac{g^{2}}{c^{2}(z)}+\frac{K g}{c^{2}(z)} \frac{d^{3} \rho_{0}}{d z^{3}} .
$$




\section{References}

1 J.S. Rowlinson and B. Widom, Molecular Theory of Capillarity (Clarendon, Oxford, 1989).

2 R. Kobayashi, "Modeling and numerical simulations of dendritic crystal growth," Physica D 63 410-423 (1993).

3 A.A. Wheeler, B.T. Murray and R.J. Schaefer, "Computation of dendrites usir shasefield model," Physica D 66 243-262 (1993).

4 J.A. Warren and W.J. Boettinger, "Prediction of dendritic growth and microsegregation patterns in a binary alloy using the phase-field method," Acta. metall. mater. 43 689$70 \bumpeq$ (1995).

${ }^{5}$ L.K. Antanovskii, "A phase-field model of capillarity," Phys. Fluids 7, 747-753 (1995).

${ }^{6}$ M.E. Gurtin, D. Polignone and J. Viñals, "Two-phase binary fluids and immiscible fluids described by an order parameter," preprint.

7 R. Chella and J. Viñals, "Mixing of a two-phase fluid by cavity flow," preprint.

${ }^{8}$ D. Jasnow and J. Viñals, "Coarse-grained description of thermo-capillary flow," preprint.

9 D. Jacqmin, "An energy approach to the continuum surface tension method," in Proceedings of the 34th Aerospace Sciences Meeting and Exhibit, (American Institute of Aeronautics and Astronautics), Reno, 1996.

10 B.U. Felderhof, "Dynamics of the diffuse gas-liquid interface near the critical point," Physica 48 541-560 (1970).

11 J.S. Langer and L.A. Turski, "Hydrodynamic model of the condensation of a vapor near its critical point," Phys. Rev. A 8 3230-3243 (1973).

12 P.C. Hohenberg and B.I. Halperin, "Theory of dynamic critical phenomena," Rev. Mod. Phys. 49 435-479 (1977).

13 B.I. Halperin, P.C. Hohenberg and E.D. Siggia, "Renormalization-group calculations of divergent transport coefficients at critical points," Phys. Rev. Lett. 32 1289-1292 (1974).

${ }^{14}$ E.D. Siggia, B.I. Halperin and P.C. Hohenberg, "Renormalization-group treatment of the critical dynamics of the binary-fluid and gas-liquid transitions," Phys. Rev. B 13 2110-2123 (1976). 
15 K. Kawasaki, "Kinetic equations and time correlation functions of critical fluctuations," Ann. Phys. 61 1-56 (1970).

16 A. Onuki and K. Kawasaki, "Nonequilibrium steady state of critical fluids under shear flow: a renormalization group approach," Ann. Phys. 121 456-528 (1979).

17 A. Onuki, K. Yamazaki and K. Kawasaki, "Light scattering by critical fluids under shear flow," Ann. Phys. 131 217-242 (1981).

18 J.U. Brackbill, D.B. Kothe and C. Zemach, "A continuum method for modeling surface tension," J. Comp. Phys. 100 335-354 (1992).

19 S.O. Unverdi and G. Tryggvason, "A front-tracking method for viscous, incompressible, multi-fluid flows," J. Comp. Phys. 100 25-37 (1992).

20 S.O. Unverdi and G. Tryggvason, "Computations of multi-fluid flows," Physica D 60 70-83 (1992).

21 R.F. Berg, M.J. Lyell, G.B. McFadden and R.G. Rehm, "Internal waves in xenon near the critical point," Phys. Fluids 8 1464-1475 (1996).

22 J.T. Ho and J.D. Litster, "Faraday Rotation near the Ferromagnetic Critical Temperature of $\mathrm{CrBr}_{3}$," Phys. Rev. B 2 4523-4532 (1970).

M.R. Moldover, J.V. Sengers, R.W. Gammon and R.J. Hocken, "Gravity effects in fluids near the gas-liquid critical point," Rev. Mod. Phys. 51, 79-99 (1979).

24 D.J. Korteweg, "Sur la forme que prennent les équations du mouvements des fluides si l'on tient compte des forces capillaires causées par des variations de densité considérables mais continues et sur la théorie de la capillarité dans l'hypothèse d'une variation continue de la densité," Arch. Néerl. Sci. Exactes Nat. Ser. II 6 1-24 (1901).

25 J.W. Cahn, "Critical Point Wetting," J. Chem. Phys. 66 3667-3672 (1977).

26 J.W. Cahn and J.E. Hilliard, "Free Energy of a Nonuniform System. I. Interfacial Free Energy," J. Chem. Phys. 28 258-267 (1958).

27 S.-L. Wang, R.F. Sekerka, A.A. Wheeler, B.T. Murray, S.R. Coriell, R.J. Braun and G.B. McFadden, "Thermodynamically-consistent phase-field models for solidification," Physica D 69 189-200 (1993).

28 G. Caginalp, "Stefan and Hele-Shaw type models as asymptotic limits of the phase-field equations," Phys. Rev. A 39 5887-5896 (1989).

29 R.J. Braun, G.B. McFadden and S.R. Coriell, "Morphological Instability in Phase-Field Models of Solidification," Phys. Rev. E 49 4336-4352 (1994). 
30 J.M. Delhaye, "Jump conditions and entropy sources in two-phase systems. Local instant formulation," Int. J. Multiphase Flow 1 395-409 (1974).

31 C.E. Weatherburn, "Differential invariants in geometry of surfaces, with applications to mathematical physics," Quarterly J. Mathematics 50 230-269 (1925).

32 H.E. Stanley, Introduction to Phase Transitions and Critical Phenomena (Oxford University Press, Oxford, 1971).

33 H.B. Callen, Thermodynamics and an Introduction to Thermostatics (Wiley, New York, 1985).

34 J. Lighthill, Waves in Fluids (Cambridge University Press, Cambridge, 1978).

35 H.B. Keller, Numerical Solution of Two Point Boundary Value Problems, Regional Conference Series in Applied Mathematics (SIAM, Philadelphia, 1976, Vol. 24).

${ }^{36}$ M.R. Scott and H.A. Watts, "Computational Solution of Linear Two-Point Boundary Value Problems Via Orthonormalization" SIAM J. Numer. Anal. 14 40-70 (1977).

37 S. Fisk and B. Widom, "Structure and free energy of the interface between fluid phases in equilibrium near the critical point," J. Chem. Phys. 50 3219-3227 (1969).

38 P.C. Hohenberg and M. Barmatz, "Gravity effects near the gas-liquid critical point," Phys. Rev. A 6 289-313 (1972).

39 R.F. Sekerka, "Notes on Entropy Production in Multicomponent Fluids," unpublished (1993).

40 C. Kittel and H. Kroemer, Thermal Physics (Freeman, New York, 1980).

41 H.S. Carslaw and J.C. Jaeger, Conduction of Heat in Solids (Clarendon, Oxford, 1959).

42 G.K. Batchelor, An Introduction to Fluid Dynamics (Cambridge University Press, Cambridge, 1967). 


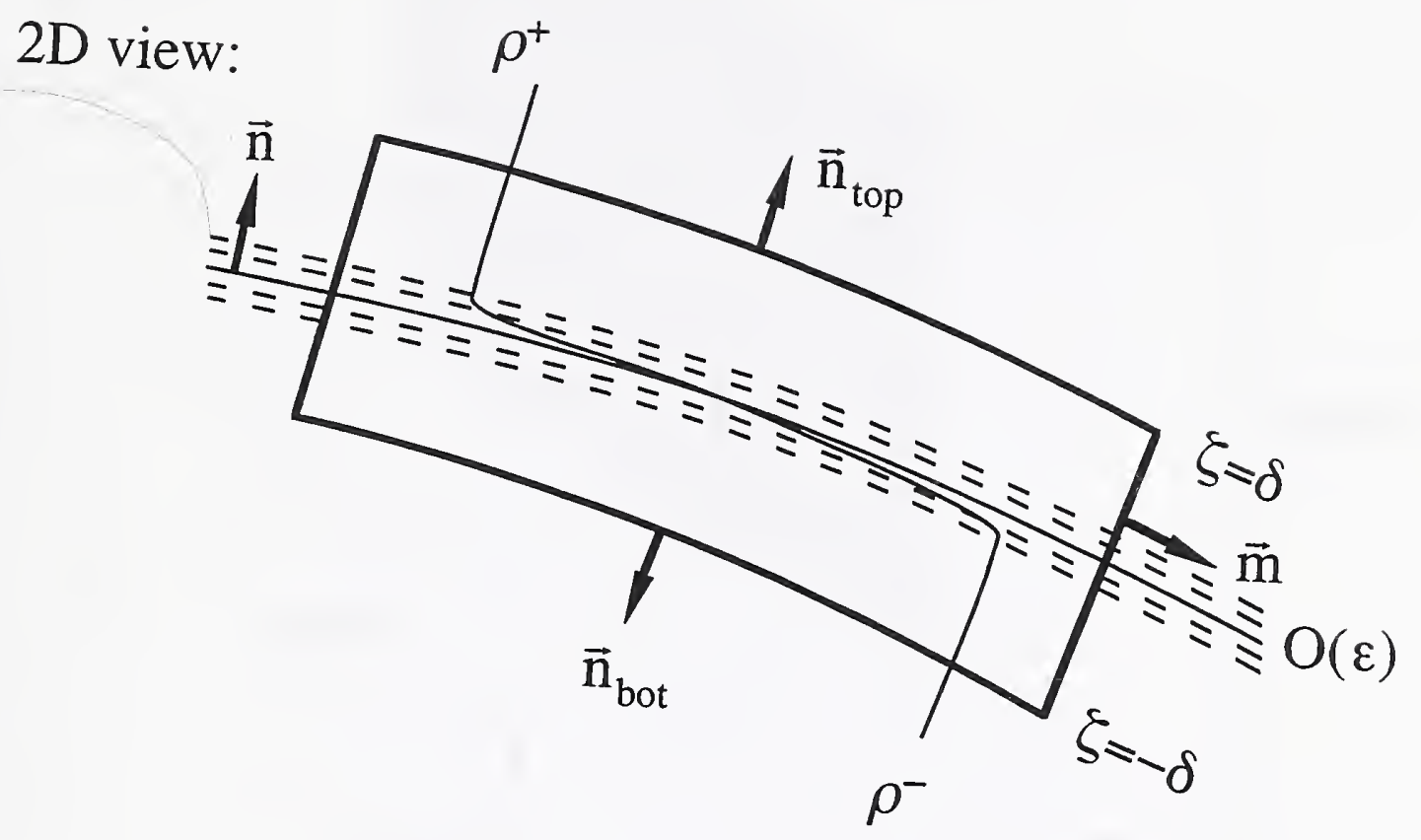

Figure 1: The pillbox enclosing the interfacial region shown in a side view. We identify the constant density surface that is the limiting surface of the interfacial region as its thickness goes to zero. This surface has the normal vector $\hat{n}$. The pillbox has normal vectors $\hat{n}_{\text {top }}, \hat{n}_{\text {bot }}$ and $\hat{m}$ on its top, bottom and side, respectively. The figure also illustrates the limiting case considered in the pillbox argument. The upper and lower surfaces of the pillbox, defined by $\zeta= \pm \delta$ where $\zeta$ is a local normal coordinate, are squeezed together in such a way that the variation in density $\rho$ through the interfacial region is completely enclosed within the pillbox. That is, we consider the limit $\epsilon \ll \delta \ll L$ where $\epsilon$ is a measure of the interfacial thickness and $L$ is an outer $O(1)$ length scale. 


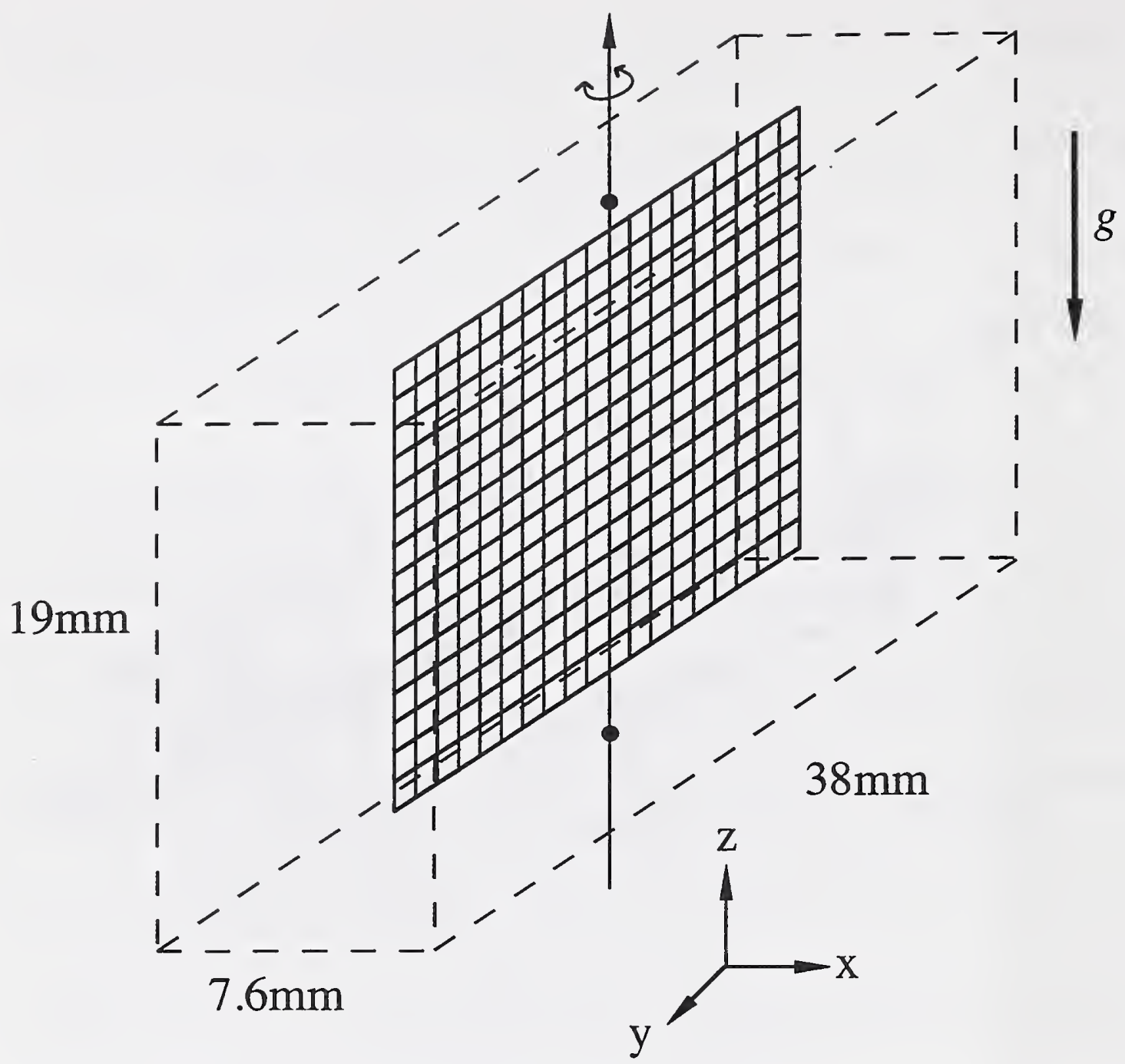

Figure 2: This figure shows the model configuration filled with a stratified fluid. The dimensions are $0 \leq x \leq a_{x}, 0 \leq y \leq a_{y}$ and $-L \leq z \leq L$ where $a_{x}=7.6 \mathrm{~mm}, a_{y}=38 \mathrm{~mm}$ and $L=9.5 \mathrm{~mm}$ are the values corresponding to the experiment by Berg et al. The paddle, which in the experiments creates the initial disturbance, is shown for reference and is not present in the mathematical model. 


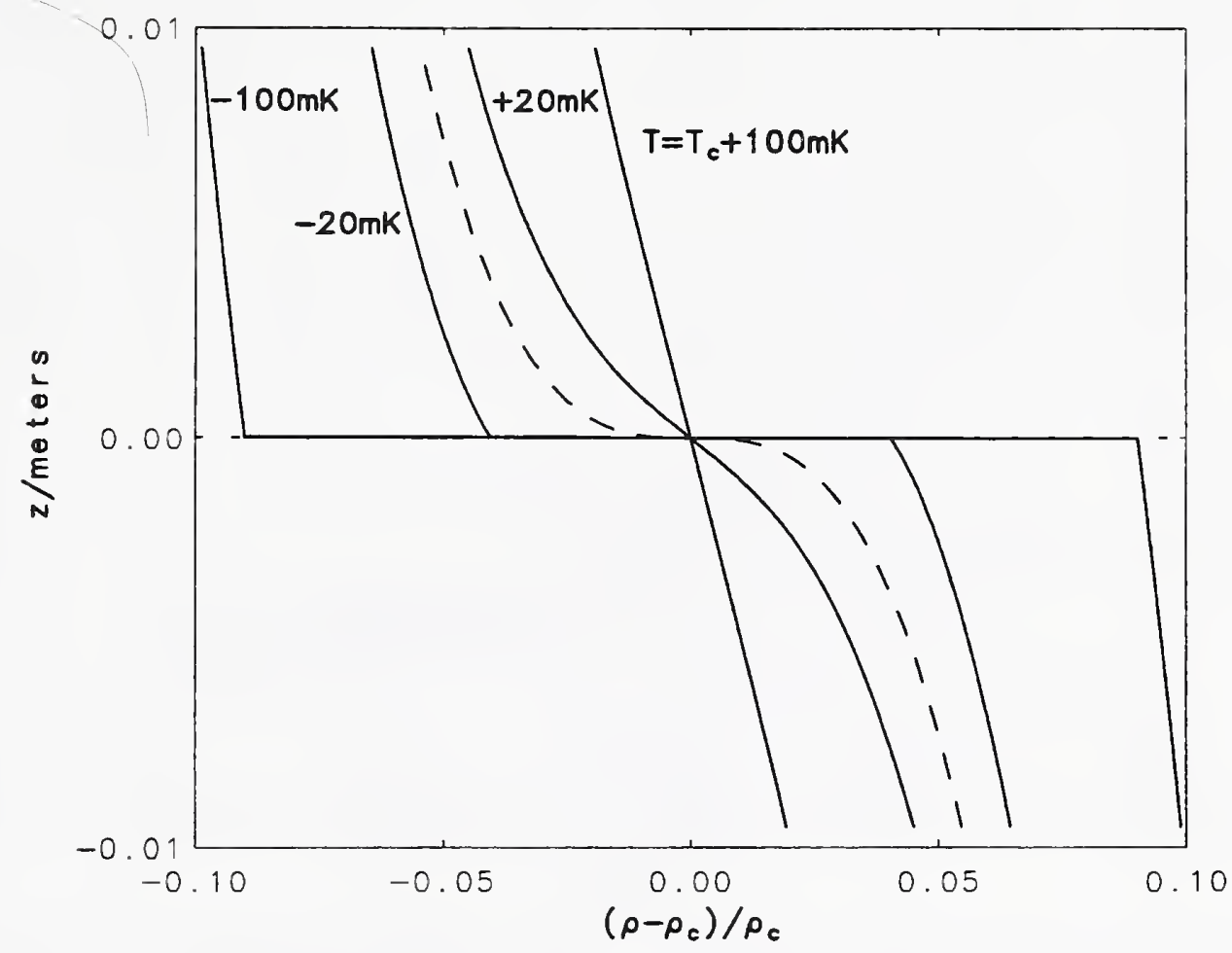

Figure 3: This figure shows the static density profiles representing solutions of equation (41). Each curve corresponds to a different temperature as indicated. The dashed curve corresponds to the density profile at the critical temperature. Above the critical temperature, there is a single stratified phase. Below the critical temperature, the fluid separates into two stratified phases. The parameter values used to calculate these profiles are $\epsilon_{D L}=10^{-4}, a=4.85$, $\tilde{g}=1.631 \times 10^{-4}$. 


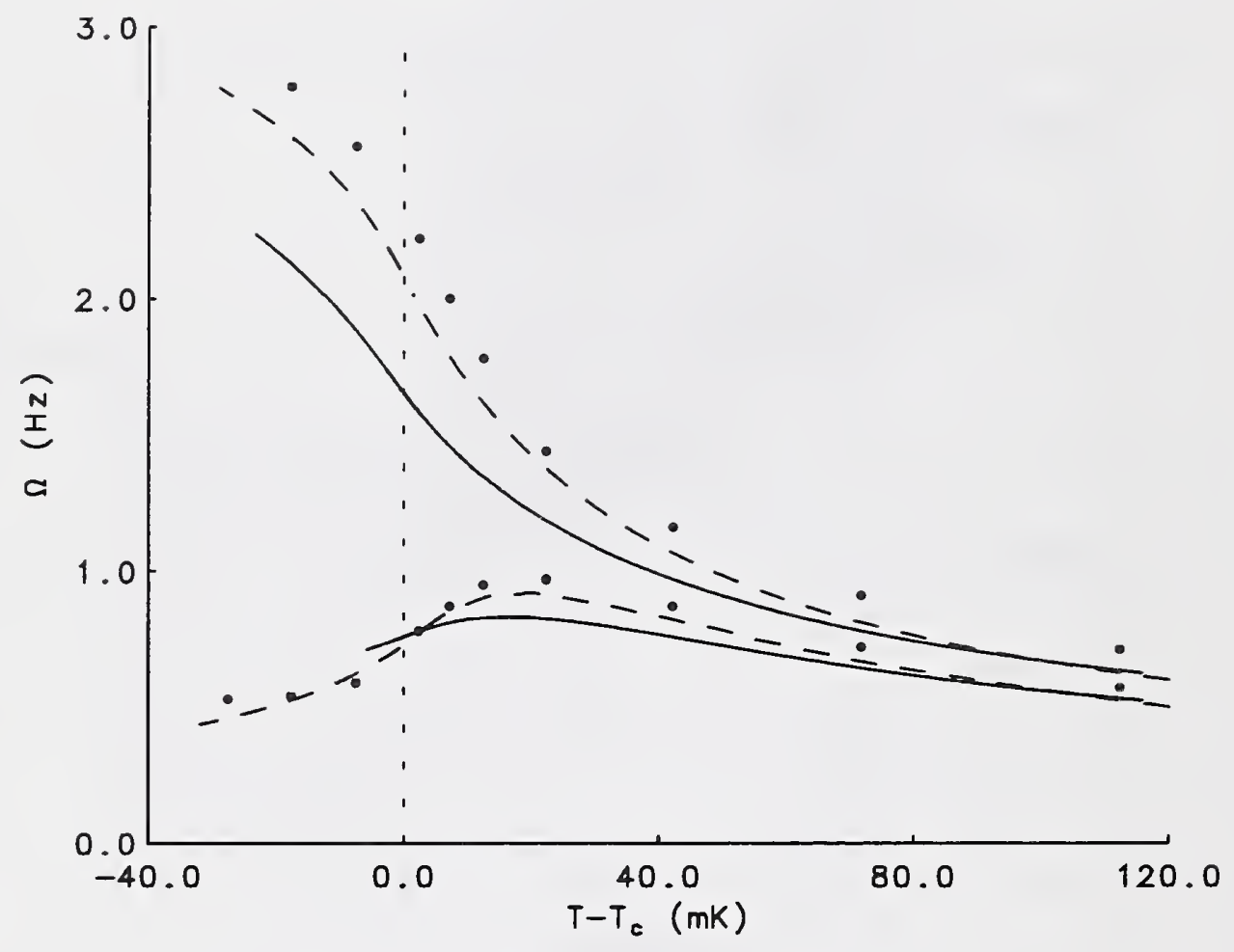

Figure 4: This figure shows the internal wave frequencies $\Omega=\omega / 2 \pi$ (in $\mathrm{Hz}$ ) obtained experimentally by Berg et al. (data points), the theoretical predictions by Berg et al. (dashed curves) using two separate models above and below the critical temperature coupled with a restricted cubic model for the equation of state, and the theoretical predictions of the present diffuse-interface approach (solid curves) using a van der Waals equation of state. The vertical dashed line indicates the critical temperature. The parameter values used for the diffuse-interface calculations are $\epsilon_{D L}=10^{-4}, a=4.85$ and $\tilde{g}=1.631 \times 10^{-4}$. 


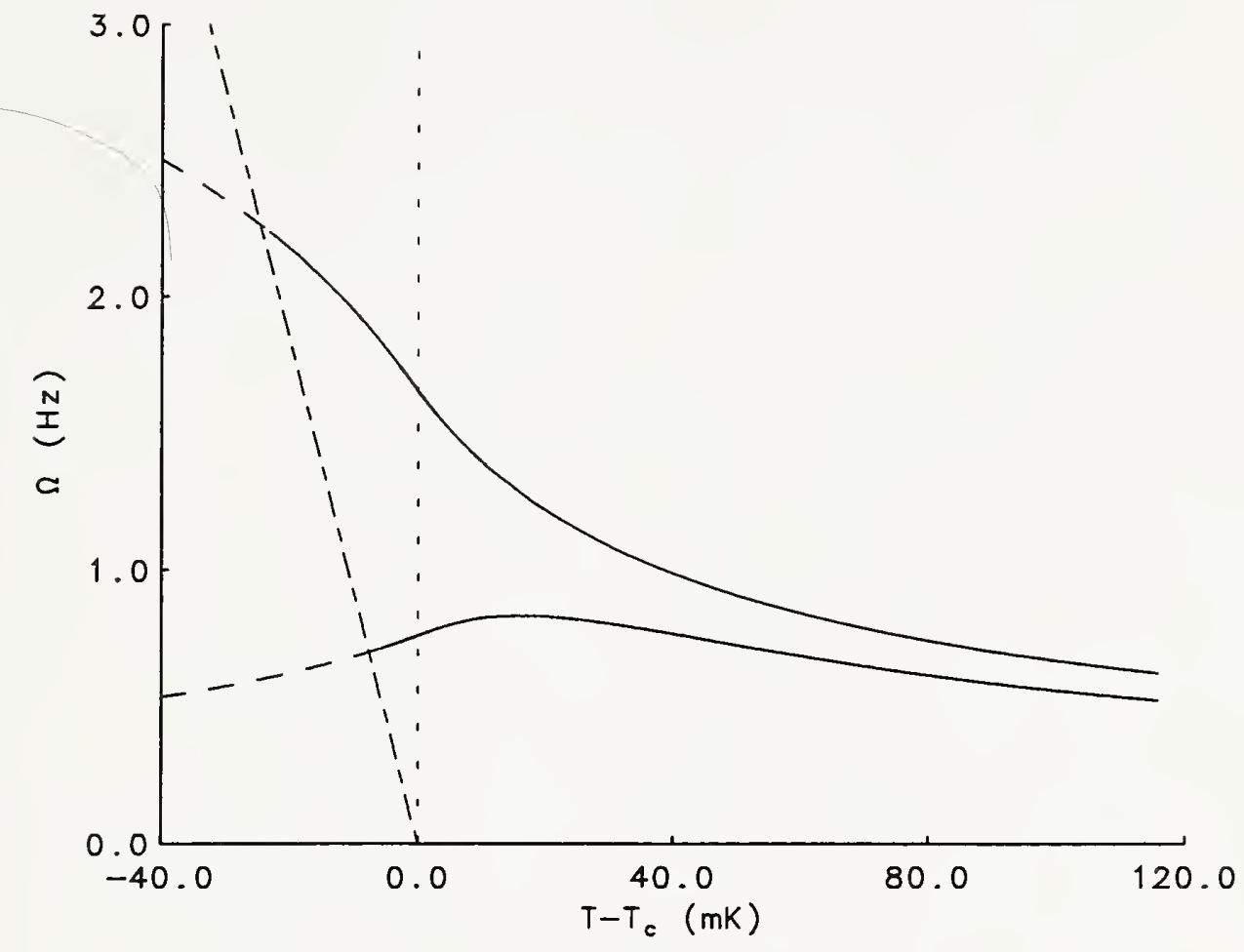

Figure 5: This figure shows a direct comparison of the frequency calculated using the classical model and that calculated with the diffuse-interface model. In each case we have used (40) as the equation of state. The vertical dashed line indicates the critical temperature. We find that in this case the diffuseinterface model (solid curves) recovers the classical model (dashed curves) given by Berg et al. The dashed-dotted line in this figure indicates the location where the coefficient $1-q^{2} M^{2} / \omega^{2}$ of the second-order term in equation (49) is predicted to vanish, and hence indicates the boundary beyond which we cannot compute with the present model in the incompressible perturbation limit. Since this coefficient depends on the vertical coordinate, we have identified this boundary by evaluating the coefficient at $\tilde{z}=0$, where the density gradient, and hence $M$, is greatest. 


